\title{
Reciprocal regulation of carbon monoxide metabolism and the circadian clock
}

\author{
Roman Klemz ${ }^{1}$, Silke Reischl ${ }^{1}$, Thomas Wallach ${ }^{1}$, Nicole Witte ${ }^{2}$, Karsten Jürchott ${ }^{1}$, Sabrina Klemz ${ }^{1}$, \\ Veronika Lang ${ }^{1}$, Stephan Lorenzen ${ }^{3}$, Miriam Knauer ${ }^{2}$, Steffi Heidenreich ${ }^{2}$, Min $\mathrm{Xu}^{4}$, Jürgen A Ripperger ${ }^{5}$, \\ Michael Schupp ${ }^{2}$, Ralf Stanewsky ${ }^{4,6}$ \& Achim Kramer ${ }^{1}$ \\ Circadian clocks are cell-autonomous oscillators regulating daily rhythms in a wide range of physiological, metabolic and \\ behavioral processes. Feedback of metabolic signals, such as redox state, NAD+/NADH and AMP/ADP ratios, or heme, \\ modulate circadian rhythms and thereby optimize energy utilization across the 24-h cycle. We show that rhythmic heme \\ degradation, which generates the signaling molecule carbon monoxide (CO), is required for normal circadian rhythms as well as \\ circadian metabolic outputs. CO suppresses circadian transcription by attenuating CLOCK-BMAL1 binding to target promoters. \\ Pharmacological inhibition or genetic depletion of $\mathrm{CO}$-producing heme oxygenases abrogates normal daily cycles in mammalian \\ cells and Drosophila. In mouse hepatocytes, suppression of CO production leads to a global upregulation of CLOCK-BMAL1- \\ dependent circadian gene expression and dysregulated glucose metabolism. Together, our findings show that $\mathrm{CO}$ metabolism \\ is an important link between the basic circadian-clock machinery, metabolism and behavior.
}

In animals, fundamental clock mechanisms are highly conserved and are based on autoregulatory feedback loops that generate molecular oscillations in the transcript levels of approximately $5-10 \%$ of genes in essentially all tissues. In mammals, the transcription-factor heterodimer CLOCK-BMAL1 (or NPAS2-BMAL1) activates the transcription of Per1, Per2 and Per3 (denoted Period) and Cry1 and Cry2 (denoted Cryptochrome) genes from E-box enhancer elements, whose protein products form complexes inhibiting CLOCK-BMAL1 transactivational activity after a delay of several hours. In additional feedback loops, CLOCK-BMAL1 also supports the rhythmic expression of Rev-Erb $\alpha$ and Rev-Erb $\beta$ (official symbols Nr1d1 and Nr1d2, respectively), which modulate the expression of Bmal1 (official symbol Arntl), and D-site-binding protein (Dbp), which modulates the expression of Per2 (ref. 1).

Although circadian-clock regulation of many metabolic processes is well established, there is increasing evidence that metabolic signals feed back to the circadian oscillator and consequently adjust metabolic pathways within individual cells ${ }^{2}$. Heme, an iron-containing porphyrin that serves as a prosthetic group in several hemoproteins, has also been described to be a ligand of REV-ERB $\alpha$. Heme binding to REV-ERB $\alpha$ supports co-repressor recruitment and transcriptional repression ${ }^{3-5}$, thereby coordinating circadian and metabolic pathways. In addition, heme binding to NPAS2 in vitro mediates the CO-sensitive DNA binding of NPAS2-BMAL1 (ref. 6).

Heme synthesis is governed by the rate-limiting enzyme aminolevulinate synthase 1 (Alas 1 ), which is negatively regulated by glucose and heme concentrations ${ }^{7}$. In addition, Alas1 transcription is circadian, through transcriptional regulation by NPAS2-BMAL1 (ref. 8). Heme catabolism is mediated by heme oxygenases 1 and 2 (Ho-1 and Ho-2; official symbols Hmox1 and Hmox2, respectively), which generate biliverdin (and subsequently bilirubin), iron and carbon monoxide at the expense of nicotinamide adenine dinucleotide phosphate (NADPH) ${ }^{9}$ Although heme can regulate the transcription of Period genes $^{8}$, and circadian heme proteins may sense the redox state and potentially diatomic gases such as oxygen, nitric oxide and $\mathrm{CO}$, the relevance of heme catabolism to circadian transcription and dynamics is unknown.

\section{RESULTS}

Inhibition of heme degradation alters circadian dynamics To test whether cellular heme levels are critical for circadian dynamics, we treated human U2-OS reporter cells expressing firefly luciferase from a Bmal1 promoter fragment with various concentrations of either hemin (iron protoporphyrin) or cobalt protoporphyrin, a specific inhibitor of the heme-degrading heme oxygenases ${ }^{10,11}$. Both compounds probably lead to increased intracellular heme levels either by providing additional heme or by inhibiting heme degradation. Whereas hemin treatment had no effect on the circadian period, the inhibition of heme degradation by cobalt protoporphyrin resulted in an inhibitor-dose-dependent lengthening of the circadian period of up to $1.5 \mathrm{~h}$ (Fig. 1). Thus, in this assay, heme-degradation products rather than the heme level itself were critical for circadian dynamics.

${ }^{1}$ Laboratory of Chronobiology, Charité Universitätsmedizin Berlin, Berlin, Germany. ${ }^{2}$ Institute of Pharmacology, Center for Cardiovascular Research CCR, Charité Universitätsmedizin Berlin, Berlin, Germany. ${ }^{3}$ Bernhard-Nocht-Institut, Hamburg, Germany. ${ }^{4}$ Department of Cell and Developmental Biology, University College London, London, UK. ${ }^{5}$ Division of Biochemistry, Department of Biology, University of Fribourg, Fribourg, Switzerland. ${ }^{6}$ Present address: Westfälische Wilhelms-Universität Münster, Münster, Germany. Correspondence should be addressed to A.K. (achim.kramer@charite.de). 
a

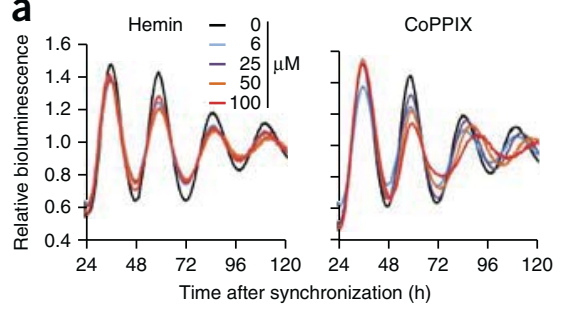

b

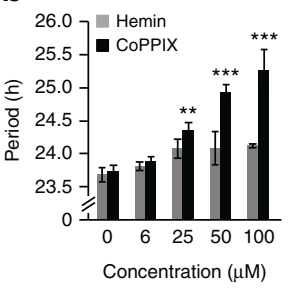

Figure 1 Inhibition of heme degradation alters circadian dynamics in human cells. (a) Circadian oscillation dynamics of dexamethasonesynchronized human osteosarcoma cells (U2-OS) bearing a Bmal1 promoter-luciferase reporter construct treated with the indicated concentrations of hemin or the heme oxygenase inhibitor cobalt protoporphyrin (CoPPIX). Shown are detrended average time series of six parallel treatments. (b) Circadian periods of time series shown in a (mean \pm s.d.; $n=6$ independent cell cultures). Two-way ANOVA with Bonferroni post test revealed a significant difference between hemin and CoPPIX effects $(P<0.0001)$ and concentration $(P<0.0001)$. Three independent experiments yielded similar results. ${ }^{* *} P<0.01 ;{ }^{* * *} P<0.001$.

\section{Heme degradation is regulated by the circadian clock}

The intricate connection between the circadian and metabolic systems manifests itself in that metabolic feedbacks into the circadian oscillator often have a circadian component. To test whether heme degradation is regulated by the circadian clock, we analyzed transcript and activity levels of $\mathrm{Ho}-1$, the major heme-degrading enzyme in peripheral tissues ${ }^{9}$. In regular 6-h intervals over the course of two consecutive days, we harvested livers from mice kept in constant darkness and determined Ho- 1 transcript levels by quantitative RT-PCR. Ho-1 expression showed a circadian rhythm with a peak at circadian time (CT) 12-15 and an approximately three-fold peak-to-trough ratio (Fig. 2a), results consistent with findings in peritoneal macrophages (Supplementary Fig. 1a) and with the diurnal variation observed by Xu and colleagues in liver tissue ${ }^{12}$.

Transcription of Ho-1 is induced by heme via stress-response enhancer elements (StREs) in its promoter ${ }^{13}$. To test whether rhythmic Ho- 1 expression is regulated by potentially rhythmic heme levels, we applied constant high concentrations of heme or cobalt protoporphyrin to synchronized primary hepatocytes. Whereas we observed the expected increase in Ho-1 transcription (and HO-1 activity, in the case of heme treatment), circadian oscillations persisted (Fig. 2b) but were absent in hepatocytes from Bmal1 ${ }^{-/-}$mice (Supplementary Fig. 1b). This finding indicates that rhythmic $\mathrm{Ho}-1$ regulation is different from rhythmic StRE activation and depends on the canonical circadian oscillator. Importantly, the activity of heme oxygenase was also rhythmic in the liver in both basal and heme-induced conditions (Fig. 2c), thus indicating that heme is rhythmically degraded. Interestingly, expression of Alas1, which encodes the rate-limiting enzyme in the heme biosynthesis pathway, is also rhythmic with a similar phase ${ }^{8}$ to that of $\mathrm{Ho}-1$, thus suggesting a potential mechanism for intracellular heme homeostasis.

To investigate whether Ho- 1 is a direct output gene of the circadian clock, we searched for clock-controlled enhancer elements within the Ho-1 promoter and identified a highly conserved $\mathrm{E}$ box close to the transcription start site (Supplementary Fig. 1c). A 1,000-bp fragment of the Ho-1 promoter was activated by CLOCK-BMAL1 in HEK293 cells and repressed by CRY1, but this regulation was abolished by E-box mutation (Fig. 2d). In addition, chromatin immunoprecipitation (ChIP) experiments on liver nuclei revealed that the Ho-1 E box was occupied by endogenous BMAL1 in a manner dependent on the time of day (Fig. 2e). Moreover, transcript levels of $\mathrm{Ho}-1$ were deceased in the hepatocytes of $\mathrm{Bmal1}^{-/-}$mice (Supplementary Fig. 1d). Together, these results support the role of $\mathrm{Ho}-1$ as a direct target gene of CLOCK-BMAL1 in vivo.

\section{The heme-degradation product $\mathrm{CO}$ modulates circadian transcription}

Rhythmic heme degradation mediated by HO-1 leads to rhythmic accumulation of heme-degradation products, i.e., biliverdin, iron and CO. In vitro, $\mathrm{CO}$ inhibits binding of NPAS2-BMAL1 to $\mathrm{DNA}^{6}$. If such an inhibitory effect also occurs in vivo, transcription of NPAS2-BMAL1target genes should increase when endogenous CO levels are decreased. Because heme oxygenase activity is the primary source of endogenous $\mathrm{CO}$, and $\mathrm{Ho}_{-} \mathrm{l}$ is the dominant paralog in the periphery ${ }^{13}$, we measured circadian transcript levels of CLOCK(NPAS2)-BMAL1-target genes in primary fibroblasts from $\mathrm{Ho}-1$-knockout mice ${ }^{14}$. Indeed, transcript levels of clock genes containing functional promoter $\mathrm{E}$ boxes, such as $D b p, R e v$-Erb $\alpha$ and Per2, were substantially upregulated, whereas transcription of Bmall was downregulated during periods in which levels of the repressor REV-ERB $\alpha$ was high (Fig. 3a). The extent of this upregulation (for example, for $D p b$ transcript levels) was somewhat variable, ranging from 1.5-fold up to six-fold (Supplementary Fig. 2). It is likely that the effect of $\mathrm{Ho}-1$ depletion on E-box-controlled mRNA levels was transcriptional rather than post-transcriptional, because precursor (pre)-mRNA levels of $D b p$ and Rev-Erb $\alpha$ were similarly upregulated in Ho-1-knockout cells (Supplementary Fig. 3a). Thus, decreasing CO levels by downregulating heme oxygenase activity has a major effect on circadian transcription.

If the upregulation of E-box-containing circadian transcripts in Ho-1-knockout animals were due a decrease in endogenous CO levels, exogenous application of $\mathrm{CO}$ should, at least in part, reverse this transcriptional effect. Continuous application of $6 \%$ gaseous $\mathrm{CO}$ (compared with 6\% nitrogen as a control for any hypoxia-induced effects) to primary fibroblasts from wild-type mice led to a slight decrease in expression of the E-box-controlled transcripts $D b p$ and Rev-Erb $\alpha$, but not Bmall (Fig. 3b). Similarly, acute CO application through use of CO-releasing molecules (CORMs) had only subtle effects on $D b p$ transcription in wild-type fibroblasts (discussion of the CO concentration in Supplementary Note 1). In contrast, acute or continuous $\mathrm{CO}$ treatment of $\mathrm{Ho}_{-1} \mathrm{I}^{-/-}$primary fibroblasts substantially, yet not always completely, rescued high $D b p$ transcript levels in Ho-1-knockout fibroblasts (Fig. 3c and Supplementary Fig. 2). Importantly, this $\mathrm{CO}$-mediated rescue was also present at the pre-mRNA level, a result consistent with the hypothesis that $\mathrm{CO}$ modulates transcription (Supplementary Fig. 3b).

\section{CO suppresses transactivation and target-gene binding of CLOCK-BMAL1}

To test whether CO-mediated transcriptional inhibition of genes containing functional $\mathrm{E}$ boxes correlates with $\mathrm{CO}$ inhibition of CLOCK(NPAS2)-BMAL1, we measured the transactivation activity of CLOCK-BMAL1 from an E-box-driven luciferase reporter in HEK293 cells treated with either CORMs or inactive controls (iCORMs). We found a CO-dose-dependent decrease in CLOCKBMAL1-mediated transcription (Fig. 4a), thus indicating that $\mathrm{CO}$ directly regulates circadian transcriptional activity. This inhibitory effect is probably due to CO interfering with CLOCK(NPAS2)BMAL1 binding to DNA, because ChIP experiments revealed that $\mathrm{CO}$ treatment decreased BMAL1 binding to the Rev-Erb $\alpha$ promoter in synchronized U2-OS cells at times when BMAL1 is transcriptionally active (Fig. 4b), although overall BMAL1 levels remained unchanged (Supplementary Fig. 3c). 

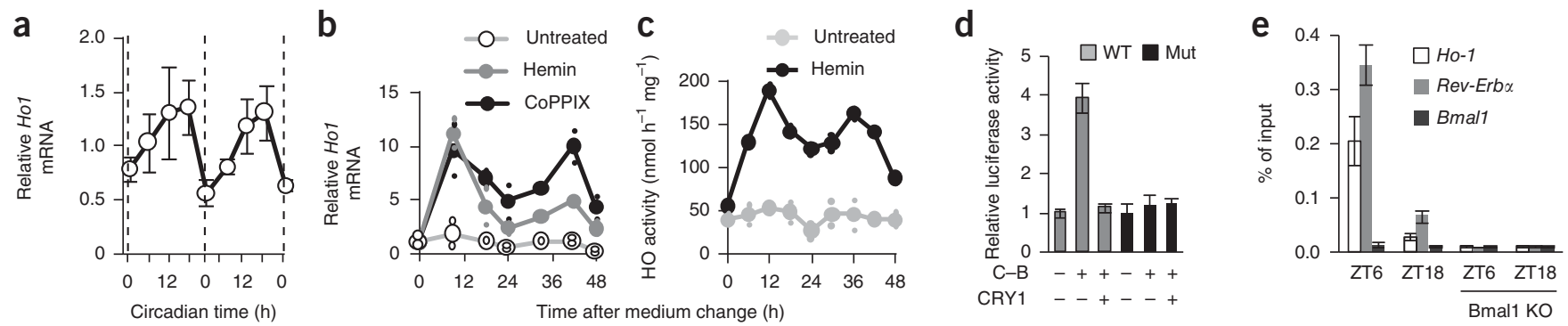

Figure 2 Heme degradation is regulated by the circadian clock. (a) Heme oxygenase 1 (Ho-1) transcript levels (normalized to Hprt and relative to mean levels) from mouse liver over $2 \mathrm{~d}$ in constant darkness (mean \pm s.e.m.; $n=4$ livers). (b) HO activity rhythms in untreated and hemin ( $30 \mu \mathrm{M}$ )-stimulated primary hepatocytes. Shown are mean levels (large symbols) of two independent hepatocyte samples (small symbols). (c) Ho-1 transcript rhythms (relative to Hprt) in primary hepatocytes treated with hemin or cobalt protoporphyrin ( $30 \mu \mathrm{M}$ each). Data are normalized to mean levels of untreated control cells. Shown are mean levels (large symbols) of two independent hepatocyte samples (small symbols). (d) CLOCK-BMAL1 (C-B)-mediated transactivation and repression by CRY1 in HEK293 cells from a Ho-1 promoter luciferase reporter with (mut) or without (WT) a mutated E box (mean \pm s.e.m.; $n=4$ independent cell cultures). (e) ChIP analysis of BMAL1 at the Ho-1, Rev-Erb $\alpha$ and Bmall genes at zeitgeber time (ZT) ZT6 and ZT18 from wild-type or Bmal1-/- $(B m a l 1 \mathrm{KO})$ liver chromatin (mean \pm s.d.; $n=6$ independent precipitations from liver samples from three mice per $Z \mathrm{ZT}$ and genotype). One-way ANOVA with a Bonferroni post test comparing all the different conditions indicated that the wild-type ZT6 data for the presence of BMAL1 at Rev-Erbo or Ho-1 were highly significantly different from all other data for the same gene, and there were no such significant changes observed for the negative control, Bmall.

Heme oxygenase-derived CO is essential for normal circadian dynamics in mammalian cells

The lengthening of the circadian period after pharmacological inhibition of heme oxygenases (Fig. 1a) suggested that heme oxygenase activity is an integral part of circadian-rhythm generation. To genetically test this phenomenon, we lentivirally transduced $\mathrm{Ho}_{-} \mathrm{I}^{-/-}$ primary fibroblasts with a Bmal1-luciferase reporter construct and measured circadian-rhythm dynamics, but we were unable to detect a change in circadian period when this heme oxygenase was individually depleted (Supplementary Fig. 4a). We therefore further decreased heme oxygenase activity (Supplementary Fig. $\mathbf{4 b}$ ), and thus probably endogenous CO levels, by RNA interference (RNAi)mediated knockdown of the second active heme oxygenase paralog, Ho-2 (ref. 15), in $\mathrm{Ho}_{-1}^{-/-}$primary fibroblasts (genotype denoted $\left.\mathrm{Ho}^{-1^{-/}} ; \mathrm{Ho}_{-} 2^{\mathrm{KD}}\right)$. Circadian rhythms in these $\mathrm{Ho}_{-1}^{-/-} ; \mathrm{Ho}^{-} \mathrm{KD}^{\mathrm{KD}}$ fibroblasts showed a substantial lengthening of the period by up to $2 \mathrm{~h}$ (Fig. 5a and Supplementary Fig. 5a), probably as a result of effects on circadian transcription, given that upregulation of the transcript
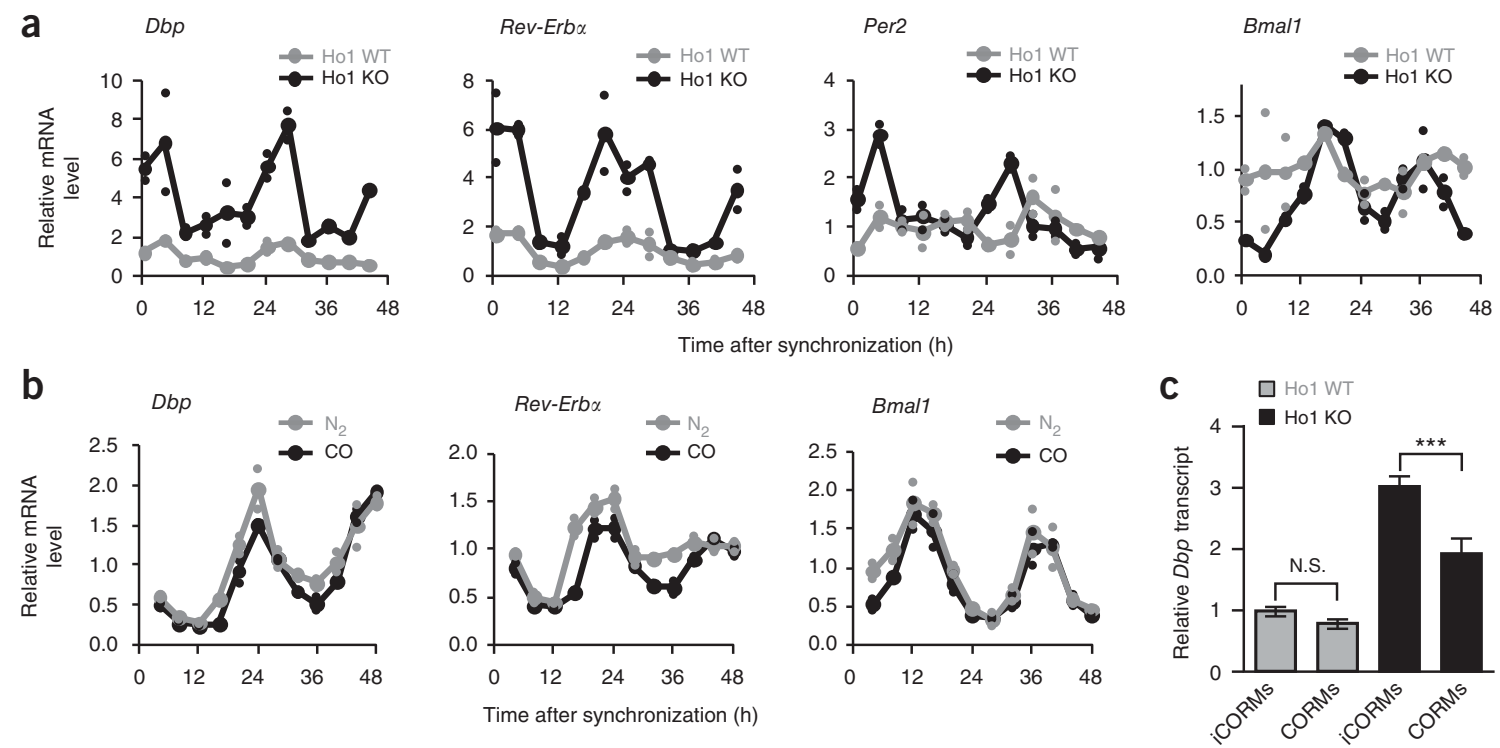

Figure 3 The heme-degradation product CO modulates circadian transcription. (a) Transcript rhythms of Bmal1, Per2, Rev-Erbo and Dbp in dexamethasone-synchronized primary fibroblasts from Ho-1-/- mice (Hol KO) or wild-type littermates (Hol WT). Data are normalized to Hprt expression and are presented relative to mean expression in wild-type cells. Shown are mean levels (large symbols) of two independent fibroblast samples from one mouse per genotype (small symbols). The amplitudes of sine fits (peak-to-trough ratios) for Hol WT versus Ho1 KO are 3.1 versus 2.9 for Dbp; 4.6 versus 6.1 for Rev-Erbo; 1.5 versus 3.2 for Per2; and 1.6 versus 3.4 for Bmal1. (b) Transcript rhythms of $D b p$, Rev-Erbo and Bmal1 in dexamethasonesynchronized primary fibroblasts from wild-type mice continuously treated with $6 \% \mathrm{CO}$ or $\mathrm{N}_{2}$. Normalization and presentation are as in a. (c) Transcript levels of $\mathrm{Dbp}$ in embryonic fibroblasts from $\mathrm{Ho}-1^{-/-}$mice (or wild-type littermates $24 \mathrm{~h}$ after dexamethasone synchronization, which were treated for $1 \mathrm{~h}$ with $100 \mu \mathrm{M}$ CO-releasing molecules (CORMs) or inactive control molecules (iCORMs) before harvesting. Data are normalized to Gapdh expression and are presented relative to mean expression in wild-type cells treated with iCORMs. Data are shown as mean \pm s.d. of three independent samples from one mouse per genotype. Two-way ANOVA with Bonferroni post test revealed a significant difference between genotypes $(P<0.0001)$ and drugs $(P<0.0001) .{ }^{* *} P<0.001 ;$ N.S., not significant. Similar experiments with samples from different mice are shown in Supplementary Figure 2. 
a

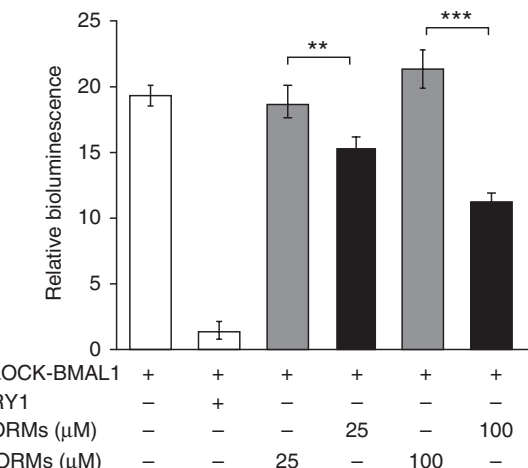

b

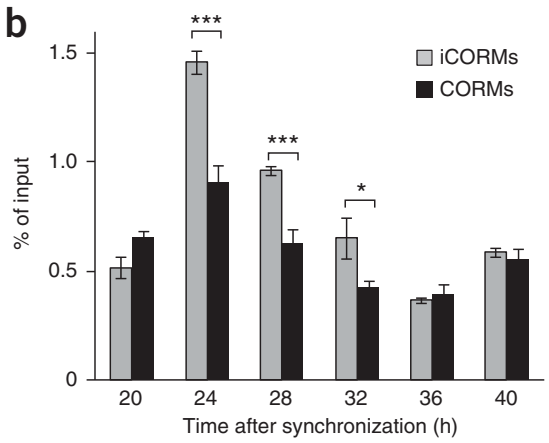

Figure 4 Carbon monoxide suppresses transactivation and target-gene binding of CLOCK-BMAL1. (a) CLOCK-BMAL1-mediated transactivation from an E-box-containing artificial promoter in HEK293 cells treated with CO-releasing molecules (CORMs) or inactive control molecules (iCORMs). Data are shown as mean \pm s.d.; $n=3$ independently transfected cell cultures. Two-way ANOVA with Bonferroni post test revealed a significant difference between iCORM and CORM treatments $(P<0.0001) .{ }^{* *} P<0.001 ;{ }^{* *} P<0.01$. (b) ChIP analysis of BMAL1 bound to the Rev-Erbo gene in synchronized U2-OS cells treated with $100 \mu \mathrm{M}$ CORMs or control iCORMs (mean \pm s.e.m. of four pairwise comparisons). Two-way ANOVA with Bonferroni post test revealed a significant difference between genotype $(P<0.0001)$ and time $(P<0.0001) .{ }^{* *} P<0.001 ;{ }^{*} P<0.05$.

levels of the E-box-controlled genes Dbp and Rev-Erb $\alpha$ was enhanced in the $\mathrm{Ho}-1^{-/-} ; \mathrm{Ho}-2^{\mathrm{KD}}$ fibroblasts (Fig. 5b).

If depletion of endogenous $\mathrm{CO}$ in $\mathrm{Ho}_{-1}^{-1-}$; $\mathrm{Ho}-2^{\mathrm{KD}}$ cells also contributes to the altered circadian dynamics, application of exogenous $\mathrm{CO}$ should be able to, at least in part, revert the period lengthening in $\mathrm{Ho}^{-1^{-/-}}$; Ho- $2^{\mathrm{KD}}$ cells. Indeed, continuous application of $6 \% \mathrm{CO}$, but not $\mathrm{N}_{2}$, led to a reshortening of the circadian period in $\mathrm{Ho}^{-1^{-1-}} ; \mathrm{Ho}-2^{\mathrm{KD}}$ fibroblasts (Fig. 5c and Supplementary Fig. 5b) but had no effect on the circadian period in wild-type cells (Supplementary Fig. 5c), thus indicating that endogenous $\mathrm{CO}$ levels are essential for normal circadian-rhythm generation.

\section{Heme oxygenase depletion globally alters clock-controlled transcription in hepatocytes}

The strong effect of heme oxygenase depletion on circadian transcription and dynamics suggests a substantial effect on clock-controlled transcriptional output. To investigate this possibility globally, we performed microarray-based genome-wide transcriptional profiling of primary hepatocytes from wild-type or $\mathrm{Ho}^{-\mathrm{-}^{-/}}$mice with or without additional knockdown of $\mathrm{Ho}-2$. As expected from results in primary fibroblasts (Fig. 3a), circadian transcript levels of the clock-controlled genes $\mathrm{Dbp}$ and $\mathrm{Rev}$-Erb $\alpha$ were upregulated in $\mathrm{Ho}^{-1^{-/-}}$hepatocytes, even more so in $\mathrm{Ho}_{\mathrm{o}} 2^{\mathrm{KD}}$ hepatocytes and to the greatest extent in
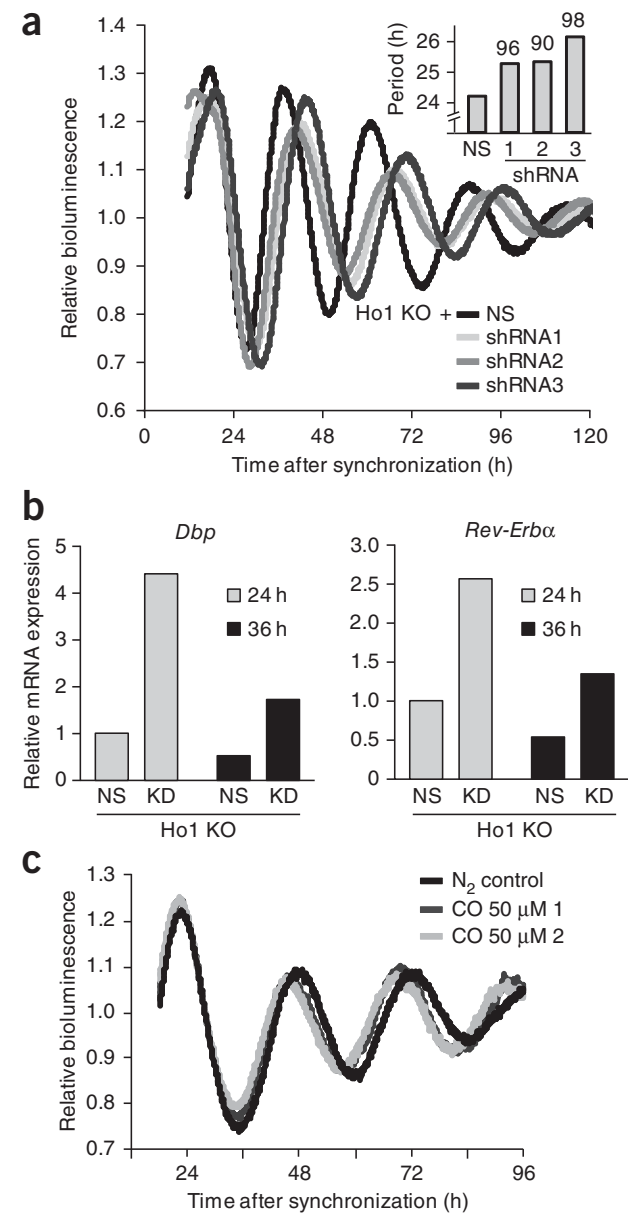

Figure 5 Heme oxygenases are essential for normal circadian dynamics in mammalian cells. (a) Circadian oscillation dynamics of synchronized primary fibroblasts from $\mathrm{Ho}-\mathrm{I}^{-/-}$mice lentivirally transduced with (i) short hairpin RNA (shRNA) constructs targeting Ho-2 or a nonsilencing (NS) control and (ii) a Bmal1 promoter-luciferase reporter construct. Shown are representative examples of detrended time series (raw data in Supplementary Fig. 5a) and period quantification (inset; numbers above bars show percentage Ho-2 mRNA knockdown). (b) Transcript levels of Dbp and Rev-Erb $\alpha 24 \mathrm{~h}$ and $36 \mathrm{~h}$ after dexamethasone synchronization of primary fibroblasts from $\mathrm{Ho}-\mathrm{I}^{-/-}$mice transduced with an shRNA construct targeting Ho-2 (KD) or a nonsilencing (NS) control. (c) Circadian dynamics of $\mathrm{HO}^{-1^{--}}$; $\mathrm{Ho}-2^{\mathrm{KD}}$ cells as in a, continuously treated with $6 \%$ $\mathrm{CO}$ or $\mathrm{N}_{2}$. Shown are two representative examples of detrended time-series (raw data in Supplementary Fig. 5b).

Ho-1-/-; Ho- $2^{\mathrm{KD}}$ cells (Fig. 6a). We identified 2,335 genes that were differentially expressed among these genotypes and grouped them into six clusters according to their gene-expression patterns (Fig. $\mathbf{6 b}$ and Supplementary Table 1).

If heme oxygenase depletion acts globally on circadian transcriptional output, genes that are rhythmically expressed in the liver should be highly represented among differentially expressed genes ${ }^{16}$. This was indeed the case in cluster $1\left(P<10^{-14}\right)$ and to a lesser extent in cluster 2 $\left(P<10^{-4}\right)$ (Fig. 6b). Cluster 1 contained 473 genes whose transcript profiles were most similar to that of the E-box-containing clockcontrolled gene Dbp (comparison with Fig. 6a). In addition, cluster 1 was highly enriched in genes reported to bind endogenous BMAL1 in ChIP experiments on liver chromatin $\left(P<10^{-22}\right.$ (ref. 17)), and genes with promoter E-box motifs (both E1 and E2 E boxes) were overrepresented only in cluster 1 (Fig. 6c). Although clusters 1 and 2 were 
a

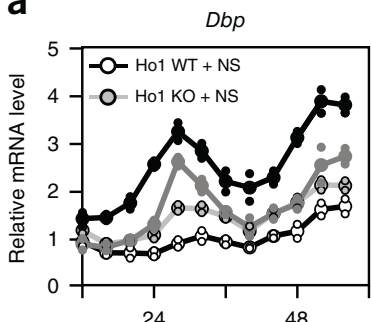

Rev-Erb $\alpha$

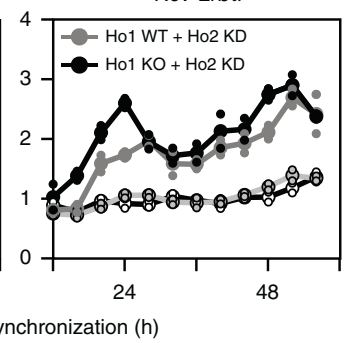

C

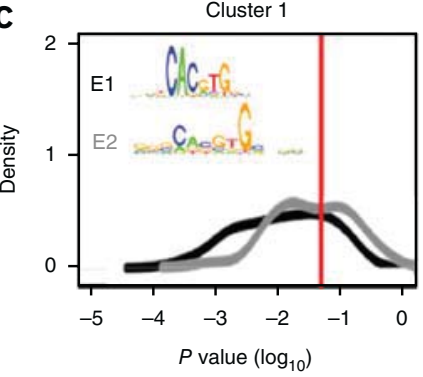

b

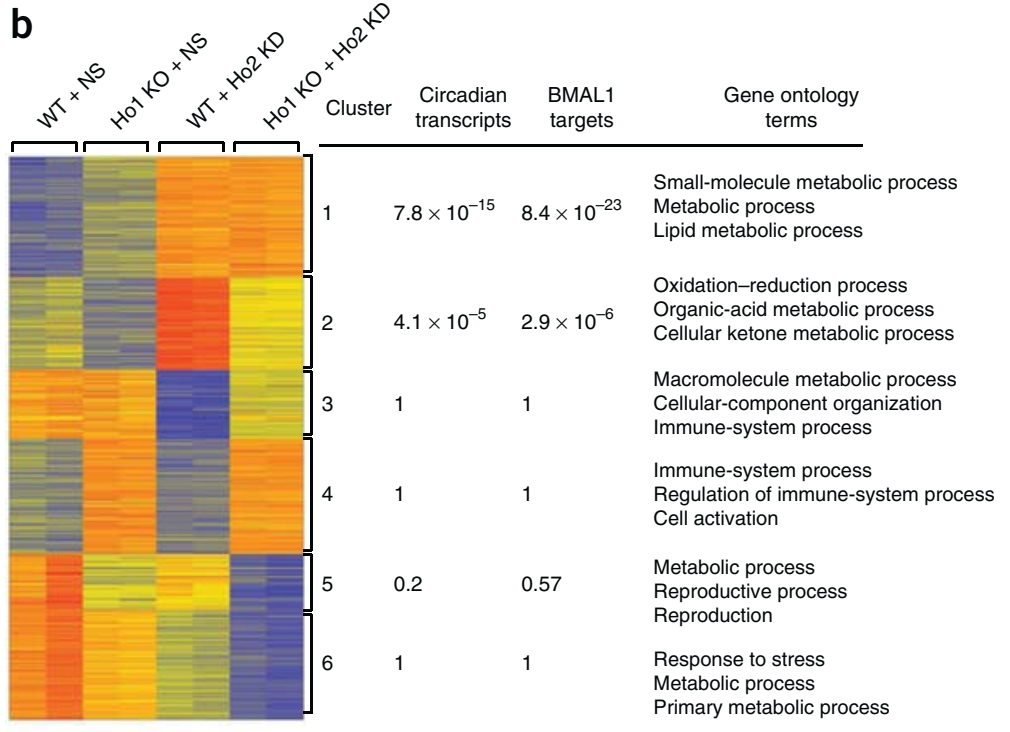

Figure 6 Heme oxygenase depletion globally alters clock-controlled transcription in hepatocytes. (a) Circadian transcription of $D b p$ and Rev-Erb $\alpha$ in synchronized primary hepatocytes of $\mathrm{Ho}^{-\mathrm{I}^{-/}}$or wild-type littermate mice with or without additional Ho-2 depletion by RNAi. Data are normalized to Gapdh expression and are presented relative to mean expression in wild-type cells transduced with the nonsilencing (NS) control. Shown are mean levels (large symbols) of two independent samples from two mice (small symbols). (b) Left, Heat diagram sorted in six clusters, showing changes in gene expression detected in a microarray experiment with hepatocytes, as described in a, $24 \mathrm{~h}$ after synchronization, the time of maximal CLOCK-BMAL1 binding to DNA ( $n=2$ mice per genotype). Increases (red) or decreases (blue) in transcript levels are shown. Middle, $P$ values indicate significance for overrepresentation (Fisher's test) of genes with a circadian transcript in liver ${ }^{16}$ or with BMAL1 binding in their promoters ${ }^{17}$. Right, Most significant nonredundant gene ontology terms for genes enriched in the clusters. (c) Bioinformatics analysis of cluster 1 genes for overrepresentation of E-box motifs in regions from $-1,000$ bp to $+1,000$ bp with respect to the transcription start site. Shown are $P$-value distributions of TRAP scores (Online Methods) for depicted E-box motifs compared with background gene sets.

also enriched in target genes of REV-ERB $\alpha$ and REV-ERB $\beta$ (ref. 18), we believe that it is unlikely that the upregulation of these targets in Ho-1- and Ho-2-depleted hepatocytes was due to CO directly regulating the activity of REV-ERB $\alpha$ and REV-ERB $\beta$ (discussion in Supplementary Note 2).

\section{Heme oxygenase depletion alters glucose homeostasis}

Gene ontology analysis revealed significant enrichment of genes involved in metabolic processes in cluster 1, whereas in the Ho-1dominated cluster 4, the significant GO terms reflect known roles of Ho-1 in cytoprotection and immune response ${ }^{13,14}$ (Fig. 6b). Genes

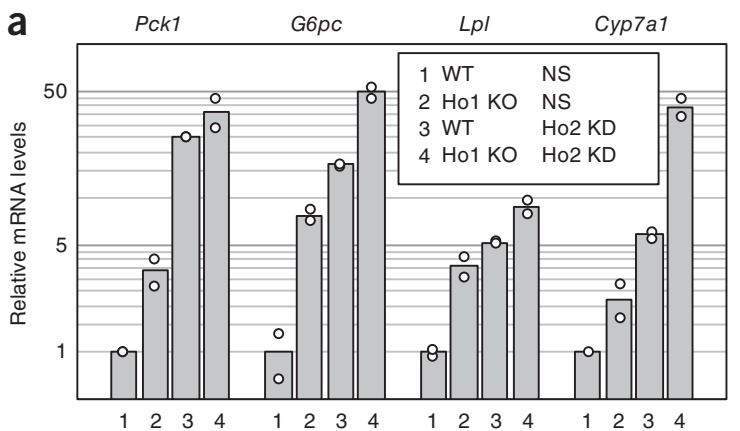

b

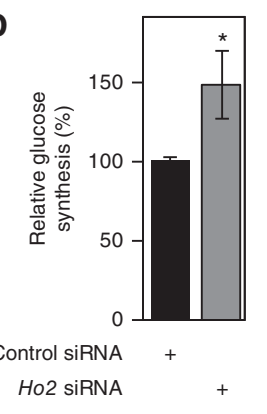

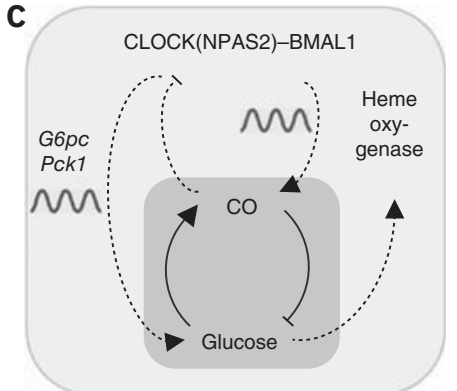

Figure 7 Interplay between carbon monoxide and glucose metabolism. (a) Transcript levels of Pck1, G6pc, Lpl and Cyp7a1 in primary hepatocytes (24 $\mathrm{h}$ after dexamethasone synchronization) of $\mathrm{Ho}_{-} \mathrm{-}^{-/-}$or wild-type littermate mice with or without additional Ho-2 depletion by RNAi. Data are normalized to Gapdh expression and are presented relative to mean expression in wild-type cells transduced with the nonsilencing (NS) control. Shown are mean levels (bars) of two independent samples from two mice (small symbols). (b) Ho-2 depletion via siRNA transfection increases glucagon-induced glucose production in hepatocytes (normalized to total protein content; mean \pm s.d.; $n=3$ independent transfections of hepatocyte cultures from the same mouse; ${ }^{*} P<0.05$ by two-tailed $t$ test). Two independent experiments yielded similar results. (c) Schematic model of the feedback interplay between endogenous $\mathrm{CO}$ and glucose homeostasis. The core of this model (dark gray) describes the activation of $\mathrm{CO}$ production by high glucose and oxidative-stress levels and the negative influence of $\mathrm{CO}$ on glucose production. The mechanisms by which these regulatory influences are exerted are depicted in the light-gray area of the scheme: endogenous $\mathrm{CO}$ is rhythmically produced by heme oxygenases, whose transcription is activated by CLOCK(NPAS2)-BMAL1 (among other factors) (Fig. 2). CO has a negative influence on CLOCK(NPAS2)-BMAL1-mediated transcriptional activity (Fig. 3 and Fig. 4), thereby also inhibiting genes important for glucose production (Fig. 7a). High glucose levels, however, lead to the induction of $\mathrm{Ho}-1$ (ref. 46) and thereby support endogenous $\mathrm{CO}$ production. 
a

dHO-RNAi:21-1/+
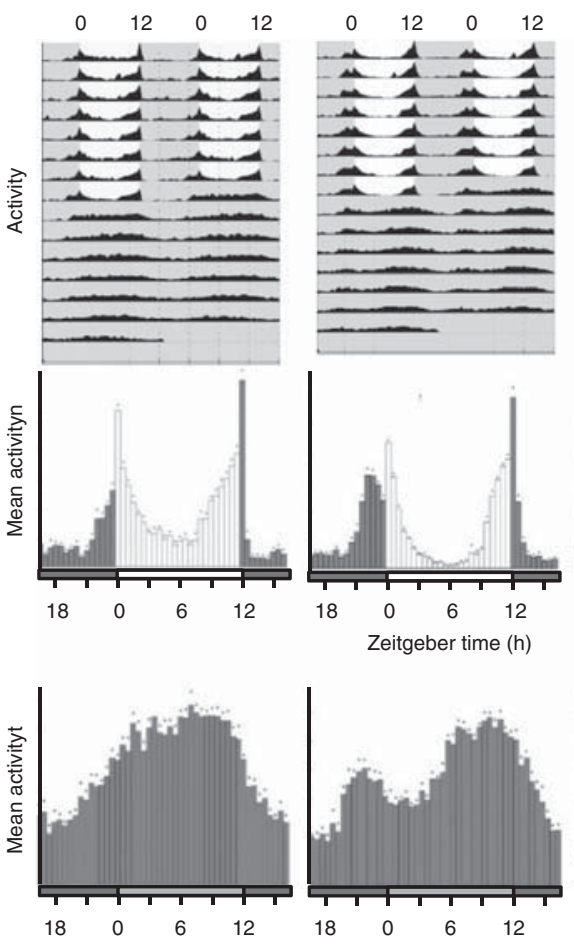

Circadian time $(\mathrm{h})$

b
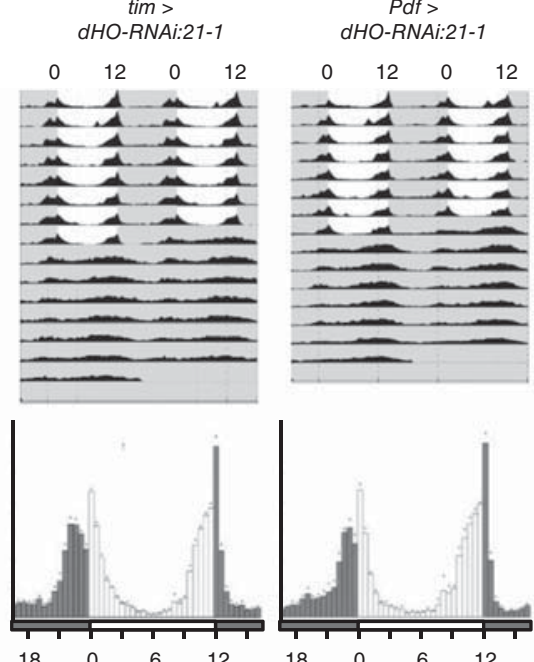

Zeitgeber time $(\mathrm{h})$

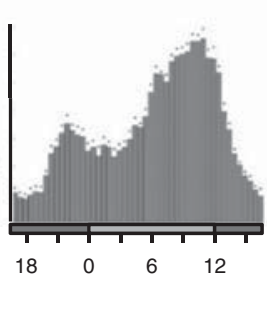

$$
\begin{aligned}
& -8.0-\text { Iuc;tim > dHO-RNAi:21-1 } \\
& -8.0-\text { Iuc;tim > GFP } \\
& - \text { - 8.0-luc;tim > UAS-51C }
\end{aligned}
$$
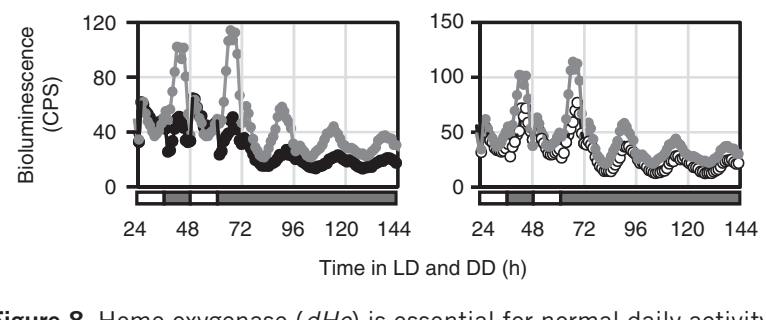

Figure 8 Heme oxygenase $(\mathrm{dHo})$ is essential for normal daily activity patterns in Drosophila. (a) Average locomotor activity of Drosophila males in $12 \mathrm{~h}$ light-12 $\mathrm{h}$ dark conditions for $5-8 \mathrm{~d}$, followed by $7 \mathrm{~d}$ in constant darkness (DD). Exact genotypes from left to right: UAS-dHo-RNAi:21-1/+ ( $n=15$ flies); timeless-gal4:62/UAS-dHo-RNAi:21-1 ( $n=16$ flies); Pdfgal4/UAS-dHo-RNAi:21-1 ( $n=16$ flies). Top, double-plotted actograms showing average activity during the entire experiment. Gray and white areas indicate dark and light periods, respectively. The morning peak in flies with decreased $d H o$ expression persists in DD but disappears rapidly in the controls. Bottom, histograms showing average activity within in $30 \mathrm{~min}$ (bars); dark bars, 'lights off'; white bars, 'lights on', dots, s.e.m. The phaseadvanced morning activity peak in flies with decreased $\mathrm{dHo}$ expression in clock cells. The same advance was observed in transgenic flies bearing a different $d H o-R N A i$ construct (additional data and additional control genotypes in Supplementary Fig. 7a). (b) Real-time luciferase recordings of flies expressing a PERIOD-LUCIFERASE fusion protein in dorsal clock neurons encoded by the promoterless 8.0 -luc transgene ${ }^{30}$. Male flies were recorded in LD and DD, as indicated by the bars below the plots (white and black bars indicate lights on and lights off, respectively). Exact genotypes: gray circles, 8.0-luc/+;tim-gal4:67/UAS-dHo-RNAi:21-1 ( $n=14$ flies); black circles, 8.0-luc/UAS-GFP; tim-gal4:67/+ ( $n=10$ flies); open circles, 8.0-Iuc/UAS-attP-51C; tim-gal4:67/+ ( $n=10$ flies). The peak levels were higher in LD and DD, and the amplitude of PER-LUC oscillations increased in $d H o-R N A i$ flies. Similar results were obtained in two independent experiments and with the dHo-RNAi:21-8 line (Supplementary Fig. 7b). important in glucose and lipid metabolism, such as $P c k 1, G 6 p c, L p l$ or Cyp7al, were strongly upregulated in $\mathrm{Ho}_{-1}^{-/-} ; \mathrm{Ho}-2^{\mathrm{KD}}$ hepatocytes and to a lesser extent in $\mathrm{Ho}_{-} \mathrm{1}^{-/-}$and $\mathrm{Ho}-2^{\mathrm{KD}}$ hepatocytes, as verified by quantitative RT-PCR (Fig. 7a). Again, heme oxygenase probably affects transcriptional rather than post-transcriptional processes, because the pre-mRNA levels of $P c k 1, G 6 p c, L p l$ and Cyp7a1 were similarly upregulated in heme oxygenase-depleted hepatocytes (Supplementary Fig. 6). Because $P c k 1$ and G6pc are important players in gluconeogenesis, we investigated the effect of acute small interfering RNA (siRNA)-mediated Ho-2 depletion on glucagon-stimulated glucose production in primary hepatocytes. Consistently with the upregulation of these enzymes in $\mathrm{Ho}$-2-depleted cells, we found a significant increase in glucagon-stimulated glucose production, thus suggesting that heme oxygenase activity is required for normal glucose homeostasis (Fig. 7b).

\section{Heme oxygenase is essential for normal daily activity patterns in Drosophila}

Because the expression of Alas1 and heme oxygenase has also been reported to be circadian in Drosophila melanogaster heads ${ }^{19,20}$, we investigated whether heme oxygenase ( $\mathrm{dHo}$ (official symbol $\mathrm{Ho}$ ), which is the only isoform of heme oxygenase in Drosophila and is essential for normal fly development ${ }^{21}$ ) is also required for normal rhythms in this species. To this end, we examined behavioral rhythms in flies in which $d H o$ expression was knocked down in various cell types by two independent UAS- $d H o-R N A i$ lines by using the binary Gal4-UAS system $^{22}$. Under standard $12 \mathrm{~h}-12 \mathrm{~h}$ light-dark (LD) cycles, wild-type flies exhibit crepuscular behavior, with pronounced morning $(\mathrm{M})$ and evening (E) activity peaks ${ }^{23}$ (Fig. 8a). When released into constant dark conditions (DD), wild-type flies maintain their E peak, whereas the M peak weakens or disappears (Fig. 8a). Knockdown of $d H o$ in all neurons (by using the pan-neuronal Gal4 driver elav-gal4) resulted in a substantially advanced $M$ peak, whereas the E peak was unaffected (Supplementary Fig. 7a). Next, we downregulated $d H o$ with the timeless (tim)-gal4 driver, which is active in all clock neurons and peripheral clock cells throughout the body ${ }^{24}$. Also, using Pdf-gal, we directed $d H o$ knockdown to the subset of the clock neurons important for regulating $\mathrm{M}$ activity ${ }^{25-27}$. In both cases, we observed a phase advance of the $M$ peak, which, interestingly, was also maintained in the subsequent DD period, thus indicating that $d H o$ knockdown in clock neurons somehow enhances M activity (Fig. 8a and Supplementary Fig. 7a). Clock cell-specific $d H o$ knockdown had no effect on E activity or circadian period in DD, and $d H o$ RNAi directed toward glia cells (by using the pan-glial driver repo-gal4) did not alter LD activity (Fig. 8a, Supplementary Fig. 7a, Supplementary Table 2 and ref. 28). To test whether heme oxygenase depletion increases transcription from E-box-containing promoters, as observed in mammalian cells, we performed a real-time in vivo luciferase reporter assay for Period transcription in live flies ${ }^{29}$. Flies in which $d H o$ was downregulated in all clock cells (tim-gal4) showed normal expression levels and temporal regulation of Period-promoter-driven luciferase expression under LD and DD conditions (Supplementary Fig. 7b). Similarly, clock cell-specific $d H O$ RNAi had no effect on the expression of a Periodluciferase transgene reporter in central and peripheral clock cells ${ }^{30}$ (Supplementary Fig. 7b). On the basis of the behavioral phenotype of $d H o$ RNAi flies, we used a reporter that directs PERIOD expression in only a subset of the behavior-controlling clock neurons and that is not active in peripheral clock cells $\left(8.0-l u c^{30}\right)$. Strikingly, and consistently with the observed strengthening of behavioral rhythms, $d H o$ knockdown resulted in a substantial increase in Period expression from the luciferase reporter during the normal peak expression times 
in LD and DD conditions, thus generating an increase in the overall amplitude of Period oscillations in LD (Fig. $\mathbf{8 b}$ and Supplementary Fig. 7b). Because the 8.0-luc construct lacks the Period promoter sequences (including the E boxes), the observed increase in PERIOD expression was probably post-transcriptionally regulated. Together, these results indicate that in Drosophila, as in mammals, the circadian system requires heme oxygenase for temporal coordination of behavioral activity and circadian accumulation of clock proteins, but in the Drosophila system, in contrast to the mammalian system, this coordination is not accomplished via increased transcription.

\section{DISCUSSION}

Here, we show that endogenous $\mathrm{CO}$ production by heme oxygenase activity is required not only for circadian oscillator function but also for balanced clock-gene and clock-target-gene expression, thereby modulating glucose homeostasis in hepatocytes. Mechanistically, we favor the hypothesis that, in mammals, heme oxygenase-derived CO attenuates CLOCK(NPAS2)-BMAL1 DNA binding, probably via coordination by a heme molecule bound to NPAS2 (ref. 6) and probably also to $\mathrm{CLOCK}^{31}$, and thereby modulates expression of CLOCK(NPAS2)BMAL1-target genes. Although this scenario is not the only possible interpretation of our results (as described below), our preferred model is consistent with both past and present observations. (i) In vitro, micromolar concentrations of CO have been shown to impair DNA binding and BMAL-heterodimer formation of heme-bound NPAS2 but not apo-NPAS2 (ref. 6), and in the brain, these concentrations of $\mathrm{CO}$ are generated by heme oxygenases ${ }^{32}$ (detailed discussion of CO concentration in Supplementary Note 1). (ii) Mutation of heme-coordinating residues within NPAS2 also impairs heterodimer formation with BMAL1, thus decreasing both specific DNA binding to canonical E boxes and NPAS2-BMAL1 transactivation activity, probably via conformational changes within the PAS-A domain of NPAS2 (ref. 33). (iii) Although much less is known about whether CLOCK is also able to bind heme and whether CO can modulate CLOCK activity, recent biophysical data have confirmed that the PAS-A domain of CLOCK binds heme in vitro with spectroscopic properties that are consistent with a sensor of diatomic gases ${ }^{31}$. (iv) We show here that depleting endogenous $\mathrm{CO}$ via heme oxygenase knockout leads to strong transcriptional upregulation of target genes of CLOCK(NPAS2)-BMAL1 in human and mouse hepatocytes. This observed modulation of circadian transcription is probably a direct effect of $\mathrm{CO}$, because application of exogenous $\mathrm{CO}$, but not $\mathrm{N}_{2}$, suppresses CLOCK-BMAL1 transcriptional activation through E-box elements, attenuates transcription of endogenous CLOCK(NPAS2)-BMAL1 targets, partially rescues transcriptional upregulation in heme oxygenase knockouts and suppresses BMAL1 binding to target promoters.

Together, these observations provide compelling evidence of a role of endogenous $\mathrm{CO}$ in modulating the transcriptional stimulatory activity of CLOCK(NPAS2)-BMAL1. However, additional potential mechanisms underlying the influence of heme oxygenases on circadian transcription and dynamics are not eliminated, because heme degradation also produces biliverdin and iron in addition to CO. Moreover, heme degradation consumes NADPH, whose intracellular concentration modulates circadian dynamics and expression of CLOCK-BMAL1target genes ${ }^{34}$. However, relevant to the present findings, we consider it unlikely that an increase in NADPH levels-as predicted to occur after heme oxygenase depletion-would generate long circadian periods and upregulation of CLOCK-BMAL1 targets, because such effects have previously been associated with decreased levels of $\mathrm{NADPH}^{34}$.

It is also possible that $\mathrm{CO}$ indirectly influences circadian gene expression. The extent of CO's effect on CLOCK(NPAS2)-BMAL1 transcriptional activity is difficult to estimate. Although CO was originally considered to be a metabolic waste product, today most of the described anti-inflammatory, antiapoptotic, antiproliferative and cytoprotective roles of heme oxygenases are ascribed to $\mathrm{CO}^{35}$. Owing to the affinity of $\mathrm{CO}$ for metal ions, the few known $\mathrm{CO}$ sensors are heme-containing proteins including hemoglobin, myoglobin, soluble guanylyl cyclase (sGC), cytochrome $c$ oxidase and the transcription factors Bach-1, Bach-2 and NPAS2. For example, heme oxygenasederived $\mathrm{CO}$ can, like nitric oxide, activate sGC, thereby leading to increased levels of cGMP. Such a mechanism has been proposed to play a role in resetting the cholinergic clock in the suprachiasmatic nucleus ${ }^{36}$, where heme oxygenase activity is circadian ${ }^{37}$. Moreover, increased cGMP levels can enhance AMP kinase (AMPK) activity ${ }^{38}$, which in turn may act on the clock by promoting CRY-protein degradation $^{39}$. Activation of AMPK in the liver also represses expression of gluconeogenesis enzymes such as $P c k 1$ and $G 6 p c^{40}$, results opposite from those observed in heme oxygenase-depleted hepatocytes. If the increases in Pck1 and G6pc transcript levels in HO-depleted hepatocytes were due to decreased AMPK activity (perhaps mediated by increased oxidative stress ${ }^{41}$ ), transcript levels of genes involved in other anabolic pathways would also be expected to be elevated ${ }^{42}$. However, this was not the case: the expression of key genes involved in lipogenesis, such as sterol regulatory element-binding protein-1, carbohydrate response element-binding protein, acetyl CoA carboxylase, fatty acid synthase, stearoyl-CoA desaturase, and glycerol-3-phosphate acyltransferase, was essentially unaltered. Thus, modulation of AMPK activity is unlikely to be the cause of altered clock function and increased gluconeogenesis in heme oxygenase-depleted cells.

Our results further establish that heme oxygenase also functions in the Drosophila circadian clock. Downregulation of $d H o$ leads to an advanced morning peak and to increased PERIOD levels and higher-amplitude oscillations in clock neurons, presumably via post-transcriptional regulation. The enhanced molecular oscillations may explain the robustness of the morning activity peak and the persistence of bimodal behavior under constant conditions. Typically, the morning activity peak weakens or disappears under constant conditions, whereas the evening peak is sustained, thus leading to unimodal behavior ${ }^{43}$. The enhanced molecular oscillations are reminiscent of the effects of the $P d f^{01}$ mutation on the same 8.0-luc reporter used in the present study ${ }^{44}$. This reporter construct is expressed in a small subset of dorsal clock neurons, and enhanced 8.0-luc oscillations in $P d f^{01}$ mutants are correlated with expression in additional dorsal clock neurons belonging to the evening oscillator, which show robust and synchronized PER oscillations in the absence of $\mathrm{PDF}^{44}$. Because we observed advanced morning activity after downregulation of $d H o$ in only the PDF cells, it is possible that heme oxygenase may affect PDF or the communication between the PDF cells and the dorsal clock neurons expressing 8.0-luc.

Interestingly, heme oxygenase activity has previously been linked to glucose homeostasis ${ }^{15}$, and $\mathrm{Ho}-2^{-/-}$mice develop symptoms of type 2 diabetes, including hyperglycemia ${ }^{45}$. However, hepatocyte $\mathrm{Ho}^{-\mathrm{I}^{-/}}$ mice are insulin hypersensitive ${ }^{46}$, and high glucose levels lead to Ho-1 induction ${ }^{47}$ as well as to increased $\mathrm{CO}$ exhalation ${ }^{48}$. Considering these results together with our present observation that heme oxygenasedepleted hepatocytes show increased gluconeogenesis, we suggest a feedback model that links CO production to the expression of genes important in glucose homeostasis (Fig. 7c). In our model, rhythmic heme oxygenase activity causes rhythmic $\mathrm{CO}$ production that in turn generates time-of-day-dependent repression of CLOCK(NPAS2)BMAL1 transcriptional activation activity. CLOCK(NPAS2)-BMAL1target genes supporting glucose production are thus inhibited by $\mathrm{CO}$, 
whereas high glucose levels lead to counter-regulation via heme oxygenase induction. Changes in redox state and heme levels not included in this feedback model are likely to have modulatory effects. It will be interesting to investigate whether $\mathrm{CO}$, as a gaseous molecule, also contributes to intercellular circadian synchronization, thereby temporally coordinating metabolic signals within the organism.

\section{METHODS}

Methods, including statements of data availability and any associated accession codes and references, are available in the online version of the paper.

Note: Any Supplementary Information and Source Data files are available in the online version of the paper.

\section{ACKNOWLEDGMENTS}

We thank A. Grudziecki, B. Koller and U. Ungethüm for excellent technical support. We also thank M. Rauer and H. Herzel for bioinformatics help as well as A. Zenclusen (Otto von Guericke University Magdeburg), M. Brunner (Ruprecht-Karls-University Heidelberg), S. Taketani (Insect Biomedical Research Center, Kyoto Institute of Technology) and the NIG-Fly Stock Center (Genetic Strain Research Center, National Institute of Genetics Mishima) for materials. This work was supported by the BBSRC (grant BB/J018589/1 to R.S.) and the German Research foundation (Emmy Noether grant SCHU 2546/1-1 to M.S. and SFB 618/A4 and SFB 740/D2 to A.K.)

\section{AUTHOR CONTRIBUTIONS}

R.K., S.R., T.W., N.W., S.K., V.L., M.K., S.H., M.X. and J.A.R. performed experiments; K.J. performed bioinformatics analyses; S.L. provided the ChronoStar software; R.K., S.R., T.W., K.J., S.K., V.L., J.A.R., M.S., R.S. and A.K. designed experiments and analyzed data; R.S. and A.K. wrote the paper; and A.K. oversaw the project.

\section{COMPETING FINANCIAL INTERESTS}

The authors declare no competing financial interests.

Reprints and permissions information is available online at http://www. nature.com/ reprints/index.html.

1. Buhr, E.D. \& Takahashi, J.S. Molecular components of the mammalian circadian clock. Handb. Exp. Pharmacol, 217, 3-27 (2013).

2. Asher, G. \& Schibler, U. Crosstalk between components of circadian and metabolic cycles in mammals. Cell Metab. 13, 125-137 (2011).

Yin, L., Wu, N. \& Lazar, M.A. Nuclear receptor Rev-erbalpha: a heme receptor that coordinates circadian rhythm and metabolism. Nucl. Recept. Signal. 8, e001 (2010).

4. Yin, L. et al. Rev-erbalpha, a heme sensor that coordinates metabolic and circadian pathways. Science 318, 1786-1789 (2007).

5. Raghuram, S. et al. Identification of heme as the ligand for the orphan nuclear receptors REV-ERB $\alpha$ and REV-ERB $\beta$. Nat. Struct. Mol. Biol. 14, 1207-1213 (2007).

6. Dioum, E.M. et al. NPAS2: a gas-responsive transcription factor. Science 298 2385-2387 (2002)

7. Oliveri, L.M., Davio, C., Batlle, A.M. \& Gerez, E.N. ALAS1 gene expression is down-regulated by Akt-mediated phosphorylation and nuclear exclusion of FOXO1 by vanadate in diabetic mice. Biochem. J. 442, 303-310 (2012).

8. Kaasik, K. \& Lee, C.C. Reciprocal regulation of haem biosynthesis and the circadian clock in mammals. Nature 430, 467-471 (2004).

9. Abraham, N.G. \& Kappas, A. Pharmacological and clinical aspects of heme oxygenase. Pharmacol. Rev. 60, 79-127 (2008)

10. Martasek, P. et al. Properties of human kidney heme oxygenase: inhibition by synthetic heme analogues and metalloporphyrins. Biochem. Biophys. Res. Commun. 157, 480-487 (1988)

11. Sardana, M.K. \& Kappas, A. Dual control mechanism for heme oxygenase: tin(IV)protoporphyrin potently inhibits enzyme activity while markedly increasing content of enzyme protein in liver. Proc. Natl. Acad. Sci. USA 84, 2464-2468 (1987).

12. Xu, Y.Q. et al. Diurnal variation of hepatic antioxidant gene expression in mice. PLoS One 7, e44237 (2012)

13. Ryter, S.W., Alam, J. \& Choi, A.M. Heme oxygenase-1/carbon monoxide: from basic science to therapeutic applications. Physiol. Rev. 86, 583-650 (2006).

14. Yet, S.F. et al. Hypoxia induces severe right ventricular dilatation and infarction in heme oxygenase-1 null mice. J. Clin. Invest. 103, R23-R29 (1999).

15. Satarug, S. \& Moore, M.R. Emerging roles of cadmium and heme oxygenase in type2 diabetes and cancer susceptibility. Tohoku J. Exp. Med. 228, 267-288 (2012).
16. Hughes, M.E. et al. Harmonics of circadian gene transcription in mammals. PLoS Genet. 5, e1000442 (2009).

17. Rey, G. et al. Genome-wide and phase-specific DNA-binding rhythms of BMAL1 control circadian output functions in mouse liver. PLoS Biol. 9, e1000595 (2011).

18. Cho, H. et al. Regulation of circadian behaviour and metabolism by REV-ERB- $\alpha$ and REV-ERB- $\beta$. Nature 485, 123-127 (2012).

19. Ceriani, M.F. et al. Genome-wide expression analysis in Drosophila reveals genes controlling circadian behavior. J. Neurosci. 22, 9305-9319 (2002).

20. Ueda, H.R. et al. Genome-wide transcriptional orchestration of circadian rhythms in Drosophila. J. Biol. Chem. 277, 14048-14052 (2002).

21. Cui, L. et al. Relevant expression of Drosophila heme oxygenase is necessary for the normal development of insect tissues. Biochem. Biophys. Res. Commun. 377 , $1156-1161$ (2008)

22. Brand, A.H. \& Perrimon, N. Targeted gene expression as a means of altering cell fates and generating dominant phenotypes. Development 118, 401-415 (1993).

23. Hamblen-Coyle, M.J., Wheeler, D.A., Rutila, J.E., Rosbash, M. \& Hall, J.C. Behavior of period-altered circadian rhythm mutants of Drosophila in light:dark cycles. J. Insect Behav. 5, 417-446 (1992).

24. Kaneko, M. \& Hall, J.C. Neuroanatomy of cells expressing clock genes in Drosophila: transgenic manipulation of the period and timeless genes to mark the perikarya of circadian pacemaker neurons and their projections. J. Comp. Neurol. 422, 66-94 (2000).

25. Grima, B., Chélot, E., Xia, R. \& Rouyer, F. Morning and evening peaks of activity rely on different clock neurons of the Drosophila brain. Nature 431, 869-873 (2004).

26. Park, J.H. \& Hall, J.C. Isolation and chronobiological analysis of a neuropeptide pigment-dispersing factor gene in Drosophila melanogaster. J. Biol. Rhythms 13, 219-228 (1998)

27. Stoleru, D., Peng, Y., Agosto, J. \& Rosbash, M. Coupled oscillators control morning and evening locomotor behaviour of Drosophila. Nature 431, 862-868 (2004).

28. Mandilaras, K. \& Missirlis, F. Genes for iron metabolism influence circadian rhythms in Drosophila melanogaster. Metallomics 4, 928-936 (2012).

29. Stanewsky, R., Jamison, C.F., Plautz, J.D., Kay, S.A. \& Hall, J.C. Multiple circadianregulated elements contribute to cycling period gene expression in Drosophila. EMBO J. 16, 5006-5018 (1997).

30. Veleri, S., Brandes, C., Helfrich-Förster, C., Hall, J.C. \& Stanewsky, R. A selfsustaining, light-entrainable circadian oscillator in the Drosophila brain. Curr. Biol. 13, 1758-1767 (2003)

31. Lukat-Rodgers, G.S., Correia, C., Botuyan, M.V., Mer, G. \& Rodgers, K.R. Hemebased sensing by the mammalian circadian protein CLOCK. Inorg. Chem. 49 , 6349-6365 (2010)

32. Ingi, T., Cheng, J. \& Ronnett, G.V. Carbon monoxide: an endogenous modulator of the nitric oxide-cyclic GMP signaling system. Neuron 16, 835-842 (1996).

33. Ishida, M., Ueha, T. \& Sagami, I. Effects of mutations in the heme domain on the transcriptional activity and DNA-binding activity of NPAS2. Biochem. Biophys. Res. Commun. 368, 292-297 (2008).

34. Rey, G. et al. The pentose phosphate pathway regulates the circadian clock. Cell Metab. 24, 462-473 (2016).

35. Kim, H.P., Ryter, S.W. \& Choi, A.M. CO as a cellular signaling molecule. Annu. Rev. Pharmacol. Toxicol. 46, 411-449 (2006).

36. Artinian, L.R., Ding, J.M. \& Gillette, M.U. Carbon monoxide and nitric oxide interacting messengers in muscarinic signaling to the brain's circadian clock. Exp. Neurol. 171, 293-300 (2001).

37. Rubio, M.F., Agostino, P.V., Ferreyra, G.A. \& Golombek, D.A. Circadian heme oxygenase activity in the hamster suprachiasmatic nuclei. Neurosci. Lett. $\mathbf{3 5 3}$, 9-12 (2003)

38. Lira, V.A. et al. Nitric oxide increases GLUT4 expression and regulates AMPK signaling in skeletal muscle. Am. J. Physiol. Endocrinol. Metab. 293, E1062-E1068 (2007).

39. Lamia, K.A. et al. AMPK regulates the circadian clock by cryptochrome phosphorylation and degradation. Science 326, 437-440 (2009).

40. Cool, B. et al. Identification and characterization of a small molecule AMPK activator that treats key components of type 2 diabetes and the metabolic syndrome. Cell Metab. 3, 403-416 (2006)

41. Shao, D. et al. A redox-dependent mechanism for regulation of AMPK activation by Thioredoxin 1 during energy starvation. Cell Metab. 19, 232-245 (2014).

42. Viollet, B. et al. AMPK: lessons from transgenic and knockout animals. Front. Biosci. (Landmark Ed.) 14, 19-44 (2009).

43. Yoshii, T., Rieger, D. \& Helfrich-Förster, C. Two clocks in the brain: an update of the morning and evening oscillator model in Drosophila. Prog. Brain. Res. 199, 59-82 (2012)

44. Yoshii, T. et al. The neuropeptide pigment-dispersing factor adjusts period and phase of Drosophila's clock. J. Neurosci. 29, 2597-2610 (2009).

45. Sodhi, K. et al. Epoxyeicosatrienoic acid agonist rescues the metabolic syndrome phenotype of HO-2-null mice. J. Pharmacol. Exp. Ther. 331, 906-916 (2009).

46. Jais, A. et al. Heme oxygenase- 1 drives metaflammation and insulin resistance in mouse and man. Cell 158, 25-40 (2014).

47. Chen, S., Khan, Z.A., Barbin, Y. \& Chakrabarti, S. Pro-oxidant role of heme oxygenase in mediating glucose-induced endothelial cell damage. Free Radic. Res. 38, 1301-1310 (2004)

48. Paredi, P., Biernacki, W., Invernizzi, G., Kharitonov, S.A. \& Barnes, P.J. Exhaled carbon monoxide levels elevated in diabetes and correlated with glucose concentration in blood: a new test for monitoring the disease? Chest 116 , 1007-1011 (1999). 


\section{ONLINE METHODS}

Mice. We used 6- to 8-week-old male C57Bl/6 mice for harvesting organs and fibroblast preparations. The animals were purchased from the Bundesinstitut für Risikobewertung (Berlin, Germany). Ho-1 ${ }^{-1-}$ mice (kind gift from A. Zenclussen) were raised with a normal diet and housed under and $12-\mathrm{h}$ light-12-h dark conditions in the Animal Facility of Charité-Universitätsmedizin, Berlin, Germany. All mouse experiments were carried out in accordance with the German and European legal and ethical regulations and were approved by the Senatsverwaltung für Gesundheit und Soziales, Berlin, Germany.

Flies. As controls, wild-type $A T D D^{49}$ and $D f 1 w(y w)$ flies were used. UAS- $d H o$ RNA $i$ line 21-1 (third-chromosome insertion), as previously described, decreases endogenous $d H o$ expression by $>50 \%$ (ref. 21 ). We obtained similar results with $U A S-d H o-R N A i$ line 21-8, bearing an independent $\mathrm{X}$-chromosomal insertion of the same construct (Supplementary Fig. 7a). To exclude any off-target effects, two insertion lines from the NIG-Fly RNAi collection (http://www.shigen.nig. ac.jp/fly/nigfly/) (UAS- $d H o-R N A i$ lines $R 1$ and $R 3$ inserted on chromosomes 3 and 2, respectively), bearing a $d H o-R N A i$ construct targeting a different region of $d H o$ RNA were tested (Supplementary Fig. 7a). As unrelated UAS controls, we used UAS-GFP:T2 (ref. 24) and an insertion of an empty pUASTattB vector into the attPlanding site at $51 C^{50}$. Pdf-gal4, tim-Gal4:62, 67, 27, repo-gal4 and elav-gal4 have been described previously ${ }^{24,51-53}$. To record period-luciferase rhythms 8.0-luc:9, XLG-luc:1 (ref. 30) and plo:3b-1I (ref. 29) were crossed into the respective mutant and control backgrounds.

Activity monitoring of flies. Locomotor activity of individual male flies aged 3 to $4 \mathrm{~d}$ was recorded automatically with a Drosophila Activity Monitoring (DAM) system (Trikinetics) as previously described ${ }^{54}$. Flies were kept in glass tubes supplemented with 5\% sucrose and $2 \%$ agar and kept under $12-\mathrm{h}-12$-h LD cycles at $25^{\circ} \mathrm{C}$ for $5-8 \mathrm{~d}$ before being released into free-running conditions (DD and $25^{\circ} \mathrm{C}$ ). Calculation of the free-running period and plotting of daily average histograms and actograms was performed with the fly toolbox and MATLab software ${ }^{55}$. Autocorrelation was applied to determine the rhythmicity and free-running period in Supplementary Table 1, as previously described ${ }^{54}$. Flies with RS (rhythmicity statistics) $>1.5$ were considered to be rhythmic. Only rhythmic flies were included in the period calculation.

Isolation and culture of primary hepatocytes. Isolation of hepatocytes was performed essentially as previously described ${ }^{56}$. In brief, livers of anesthetized 6- to 12-week-old male WT C57BL/6J or $\mathrm{Ho}^{-1^{-/-}}$mice were perfused with digestion buffer containing 5,000 U collagenase (Worthington Biochemical). After filtration and separation with a Percoll gradient (Biochrome), cells were seeded in gelatin ( $0.2 \%$ in PBS) or collagen-coated 12-well plates in Dulbecco's modified Eagle's medium (DMEM) containing 10\% FBS (Invitrogen) and 1\% penicillin/ streptomycin (Gibco).

Isolation and culture of primary fibroblasts. 6- to 12-week-old male mice were sacrificed, and ear, lung or tail tissues were cut into very small pieces and incubated overnight in cell culture medium (DMEM supplemented with 20\% FBS, penicillin/streptomycin and HEPES and amphotericin B (final concentration $2.5 \mu \mathrm{g} / \mathrm{ml}$ )) mixed with Liberase (Roche). The next day, cells were pelleted by centrifugation, seeded into a new dish and grown in medium containing $20 \%$ FBS and amphotericin B for $4 \mathrm{~d}$. Then culture conditions were changed to normal cell culture medium containing 10\% FBS and no amphotericin B. Cells were either frozen in liquid nitrogen or used directly in experiments.

Synchronization of circadian rhythms. Circadian rhythms were synchronized by treatment with $1 \mu \mathrm{M}$ dexamethasone for 30-60 min.

RNA interference. Experiments with shRNA. Hairpin shRNAs in the pLKO.1 lentiviral vector designed by The RNAi Consortium (TRC) for mouse Ho-2 were purchased from Thermo Scientific, OpenBiosystems. The following clone IDs were used: TRCN0000076353 (mature antisense, ATGTGAAGTAAAGTGCAGTGG), TRCN0000076356 (mature antisense, ATAGCTCCTTCTTAATGTTTC) and TRCN0000076357 (mature antisense, TGTAACGAGTATAAGCATGGG). As a nonsilencing control, a pLKO.1 vector containing scrambled shRNA (plasmid 1864, Addgene, hairpin sequence CCTAAGGTTAAGTCGCCCTCGCTC
GAGCGAGGGCGACTTAACCTTAGG) was used. For lentivirus production, HEK293T cells (Thermo Scientific HCL4517, regularly tested for mycoplasma) were seeded in T25 flasks and transfected with $2.5 \mathrm{mg}$ psPAX, $1.5 \mathrm{mg}$ pMD2G and $3.5 \mathrm{mg}$ of the respective lentiviral expression plasmid (RNAi constructs) with a CalPhos Mammalian Transfection Kit (BD Biosciences) according to the manufacturer's protocol. Virus-containing supernatants were filtered, and cells were transduced with $1 \mathrm{ml}$ of virus filtrate plus $8 \mathrm{ng} / \mathrm{ml}$ protamine sulfate (Sigma). After $24 \mathrm{~h}$, medium was exchanged to puromycin-containing $(10 \mu \mathrm{g} / \mathrm{ml})$ medium for selection.

Experiments with siRNA. After the attachment of hepatocytes to the cell culture plate, the medium was replaced with $500 \mu \mathrm{l}$ of DMEM only, and cells were transfected with $1 \mathrm{nM}$ of siRNA (Eurogentec) and $4 \mu \mathrm{l}$ of Lipofectamine 2000 (Invitrogen) per well overnight. The next morning, the medium was replaced by DMEM containing $10 \%$ FBS and 1\% penicillin/streptomycin.

Protoporphyrin preparation. For hemin $\left(\mathrm{Fe}^{3+}\right.$-protoporphyrin IX) and cobaltprotoporphyrin IX (Sigma-Aldrich), stock solutions of $1 \mathrm{mg} / \mathrm{ml}$ were prepared: $10 \mathrm{mg}$ porphyrin was dissolved in $800 \mu \mathrm{l} \mathrm{NaOH}(0.2 \mathrm{M})$ and incubated for $30 \mathrm{~min}$ in darkness (RT). $8 \mathrm{ml}$ PBS was added and the $\mathrm{pH}$ was adjusted to 7.4 with $150-200 \mu \mathrm{l}$ of $1 \mathrm{M} \mathrm{HCl}$.

Transfection and luciferase reporter assays. Transient transfections were carried out with Lipofectamine 2000 (Life Technologies) according to the manufacturer's protocol. HEK293 cells (human, ATCC CRL-1573, regularly tested for mycoplasma) were seeded in 24-well plates in antibiotic-free medium. Transfection was performed after cells had reached $80-90 \%$ confluence. The total amount of transfected DNA in each sample was $1.2 \mu \mathrm{g}$, composed of a firefly luciferase reporter construct (50 ng pGL3/mouse Ho-1_E-box_WT or 50 ng pGL3/mouse Ho-1_E-box_mut) and mouse Clock (300 ng), mouse Bmall (300 ng) and mouse Cry1 (30 ng), as indicated. For normalization, $2 \mathrm{ng}$ of a Renilla luciferase vector pRL-SV40 was cotransfected. The total amount of DNA per well was adjusted to $1.2 \mu \mathrm{g}$ by addition of empty pDEST26 vector. Cells were harvested $48 \mathrm{~h}$ after transfection in $200 \mu \mathrm{l}$ passive lysis buffer (PLB) and frozen at $-80^{\circ} \mathrm{C}$ for $1 \mathrm{~h}$. Cell lysates were homogenized by vortexing, and luciferase activity was measured with a Dual-Luciferase Reporter Assay System (Promega) according to the manufacturer's protocol, by using a 96-well-plate-reading luminometer (Orion II, Berthold Detection System). $5 \mu$ lof each cell extract was measured in duplicate by the addition of $25 \mu \mathrm{L}$ LARII to measure firefly luciferase activity and then $25 \mu \mathrm{l}$ Stop \& Glo reagent to detect the Renilla luciferase activity. For data analysis, firefly luciferase activity was normalized to the corresponding Renilla luciferase activity.

Cloning and mutagenesis. The $-1,000$-bp region of the mouse $\mathrm{Ho}-1$ promoter was cloned into the luciferase reporter vector pGL3prom (Promega) by use of the $\mathrm{KpnI}$ and NheI restriction sites. The resulting luciferase reporter vector containing an E box (pGL3/mouse Ho-1_E-box_WT) was verified by DNA sequencing. Mutagenesis of the E box to an inactive form (CACGTG to TTTAGT; described in ref. 57) was performed by site-directed mutagenesis (QuikChange Site-Directed Mutagenesis Kit; Stratagene).

Live-cell and fly bioluminescence recording. U2-OS cells (human, ATCC HTB-96, regularly tested for mycoplasma) stably expressing firefly luciferase from a $0.9-\mathrm{kb}$ Bmall promoter fragment ${ }^{58}$ were seeded into a white 96 -well plate $\left(20 \times 10^{4}\right.$ cell/well). After $24 \mathrm{~h}$, cells were synchronized with a single pulse of dexamethasone $(1 \mu \mathrm{M})$ for $30 \mathrm{~min}$. After being washed with PBS, cells were cultured in $150 \mu \mathrm{l}$ phenol red-free DMEM containing $10 \%$ FBS, antibiotics (100 U/ml penicillin and $100 \mu \mathrm{g} / \mathrm{ml}$ streptomycin), and $250 \mu \mathrm{M} \mathrm{D}$-luciferin (Biothema). Bioluminescence recordings were performed at $35-37{ }^{\circ} \mathrm{C}$ in a 96-well-plate-reading luminometer (TopCount, PerkinElmer). Primary fibroblasts were lentivirally transduced with reporter vector expressing firefly luciferase from a 0.9-kb Bmall promoter fragment ${ }^{58}$. Bioluminescence rhythms were analyzed in light-tight boxes with a single photomultiplier tube (Hamamatsu Photonics). Raw data were detrended through division by the 24 -h running average. Periods were estimated by fitting the cosine wave function with ChronoStar software, as described in ref. 58.

Luciferase expression of individual flies carrying the various period-luciferase transgenes were measured as described in ref. 30. Briefly, 2- to 3-d-old males were ether-anesthetized and loaded in a 96-well plate in which every other well 
contained $100 \mu \mathrm{l}$ of $5 \%$ sucrose, $1 \%$ agar and $15 \mathrm{mM}$ luciferin (Biosynth). Flies were measured in a TopCount instrument (PerkinElmer) for $3 \mathrm{~d}$ in $12-\mathrm{h}-12-\mathrm{h}$ $\mathrm{LD}\left(25^{\circ} \mathrm{C}\right)$ followed by DD for additional $3 \mathrm{~d}$. Raw data were plotted with BRASS software (version 2.1.3) (ref. 59).

Western blotting. U2-OS cells were seeded in $10 \mathrm{~cm}^{2}$ dishes. On the next day, the cells were synchronized by addition of $1 \mu \mathrm{M}$ dexamethasone for $20 \mathrm{~min}$. $23 \mathrm{~h}$ after synchronization, the cells were treated with either CORMs (dissolved in DMSO) or iCORMs (dissolved in ethanol) $(100 \mu \mathrm{M}$ final concentration, solvent was adjusted in all dishes) for $1 \mathrm{~h}$. For harvesting, cells were washed with PBS, lysed in RIPA buffer ( $1 \times$ PBS, 1\% IGEPAL CA-630, 0.5\% Na-deoxycholate and $0.1 \%$ SDS) containing protease-inhibitor cocktail (Sigma-Aldrich). Lysates were homogenized by passage $5-10$ times through a 21 -gauge syringe needle and then centrifuged. The protein concentrations of the supernatants were determined with BCA assays. $25 \mu \mathrm{g}$ of total protein was denatured in NuPAGE LDS loading buffer (Life Technologies) by boiling for $5 \mathrm{~min}$ at $95{ }^{\circ} \mathrm{C}$, separated by SDS-PAGE with NuPAGE 4-12\% Bis-Tris gels (Life Technologies) according to the manufacturer's protocol, transferred to a nitrocellulose membrane and incubated with an anti-BMAL1 primary antibody (gift from M. Brunner; validated in ref. 17) and anti- $\beta$-actin (A3853, Sigma-Aldrich). The next day, membranes were probed with HRP-conjugated secondary antibodies (sc-2317, Santa Cruz Biotechnologies), and a chemiluminescence assay was performed using Super Signal West Pico substrate (Pierce) followed by protein detection with a ChemoCam Imager (INTAS)

Determination of HO activity. HO activity was determined as previously described ${ }^{60}$. Briefly, lysates of cultured cells were prepared by mixture with lysis buffer (100 mM Tris/ $\mathrm{HCl}, 150 \mathrm{mM} \mathrm{NaCl}$ and $1 \%$ Triton X-100, pH 7.4) and analyzed for the amount of total protein with Coomassie blue. The reactions were performed with $10 \mu \mathrm{l}(20-100 \mu \mathrm{g}$ total protein) of the crude lysates in $100 \mathrm{mM}$ Tris- $\mathrm{HCl}$ with $15 \mu \mathrm{M}$ hemin, $0.8 \mathrm{mM}$ NADPH, $1 \mathrm{mM} \mathrm{MgCl}_{2}, 0.8 \mathrm{mM}$ glucose6-phosphate, $300 \mu \mathrm{M}$ BSA and $1 \mathrm{U}$ glucose-6-phosphate dehydrogenase and $3 \mu \mathrm{l}$ of a bacterial lysate of biliverdinreductase (BVR)-overexpressing cells. The reaction samples were mixed, and $100 \mu \mathrm{l}$ of the mixture was transferred into a black 96-well fluorescence microtiter plate (Nunc) in triplicates. Fluorescence emission was detected at a wavelength of $528 \mathrm{~nm}( \pm 2 \mathrm{~nm})$ in a fluorescence reader (Infinte M200, Tecan) at $37^{\circ} \mathrm{C}$ with excitation at $441 \mathrm{~nm}$, with a gain value of 140 . Fluorescence was monitored every $3 \mathrm{~min}$ over $1.5 \mathrm{~h}$ and was detected five times per well and time point. The arithmetic mean was used to calculate the slope of the linear increase in fluorescence. $\mathrm{HO}$ activities were determined on the basis of an empirical value of $0.14 \mathrm{pmol}$ bilirubin production per unit of fluorescence.

Chromatin immunoprecipitation (ChIP). ChIP from liver tissue from male wild-type (C57Bl/6) or Bmal1 ${ }^{-1-}$ mice (Fig. 2f) was performed as previously described ${ }^{61}$. Primers for the E-box-containing region in the mouse Ho-1 promoter were: $5^{\prime}$-TGCAACAGCAGCGAGAAC-3' (forward), 5'-GAAACTC TGGAGGCGACTG-3' (reverse) and 5'-FAM-CCACCACGTGACCCGCGTACTAMRA- $3^{\prime}$ (probe). As a positive control for BMAL1 binding, we used the upstream region of Rev-Erb $\alpha$ with 5'-TCACATGGTACCTGCTCCAG-3' (forward), 5'-CTTTTGCCCGAGCCTTTC-3' (reverse) and 5'-FAM-ACAGAG GGCTCTGCGCAGGC-TAMRA-3' (probe); as a negative control, we used the promoter region of Bmall with $5^{\prime}$-AGCGAGCCACGGTGAGTGT-3' (forward), $5^{\prime}$-CCGGGAACTCGCGACCC- $3^{\prime}$ (reverse) and 5'-FAM-AGCC GTCTCGGGGCGTCCCG-TAMRA-3' (probe). For carbon monoxide treatment (Fig. 4b), U2-OS cells were grown in $15-\mathrm{cm}$ dishes to $95 \%$ confluence. Cells were synchronized with $1 \mu \mathrm{M}$ dexamethasone for $1 \mathrm{~h}$ and treated with either $100 \mu \mathrm{M}$ CORMs or iCORMs for $1 \mathrm{~h}$ before cross-linking at the described time points. Cross-linking, chromatin preparation and chromatin immunoprecipitation were performed as described in ref. 61, with modifications. Cells were sonicated on ice for $15 \mathrm{~s}$ five times at a $50 \%$ setting, then centrifuged for $10 \mathrm{~min}$ $(16,000 \mathrm{~g})$. Supernatants were diluted in buffer $(1.1 \%$ Triton X-100, 2 mM EDTA, $150 \mathrm{mM} \mathrm{NaCl}$ and $20 \mathrm{mM}$ Tris- $\mathrm{HCl}, \mathrm{pH}$ 8.1) and incubated with anti-BMAL1 antibody ${ }^{13}$ (a kind gift from M. Brunner), for $1 \mathrm{~h}$ under rotation (RT); agarose-A bead slurry in the same buffer was then added, and samples were incubated for $1 \mathrm{~h}$ under rotation (RT). Precipitates were washed sequentially in TSE I $(0.1 \%$ SDS, $1 \%$ Triton X-100, 2 mM EDTA, $20 \mathrm{mM}$ Tris-HCl, pH 8.1, and $150 \mathrm{mM}$ $\mathrm{NaCl})$, TSE II (0.1\% SDS, $1 \%$ Triton X-100, 2 mM EDTA, 20 mM Tris- $\mathrm{HCl}$,
pH 8.1, and $500 \mathrm{mM} \mathrm{NaCl})$, TSE III ( $0.25 \mathrm{M} \mathrm{LiCl}, 1 \% \mathrm{NP}-40,1 \%$ deoxycholate, $1 \mathrm{mM}$ EDTA and $10 \mathrm{mM}$ Tris- $\mathrm{HCl}$, $\mathrm{pH}$ 8.1) and TSE IV (10 mM Tris-HCl, $\mathrm{pH} 8.1,150 \mathrm{mM} \mathrm{NaCl}$ and $1 \mathrm{mM}$ EDTA). Cross-linking was reversed at $65^{\circ} \mathrm{C}$ in TSE V (20 mM Tris-HCl, pH 7.5, $150 \mathrm{mM} \mathrm{NaCl}, 2 \mathrm{mM}$ EDTA and 1\% SDS) overnight. DNA fragments were purified with a QIAquick Spin Kit (Qiagen) and eluted in $60 \mu \mathrm{l}$ TE buffer. Q-PCR was performed with SYBR Green (Fermentas) on a CFX384 Real-Time PCR Detection System (Bio-Rad). For detection of BMAL1 binding to an E box in the promoter region of the human Rev-erbo gene, the primers $(1 \mu \mathrm{M}) 5^{\prime}$-CCTTCTCTGGACTTTGCCCT- 3 ' (forward) and 5'-AAACCTTGCAAACGTGAGGG-3' (reverse) were used.

Real-time quantitative PCR. Total RNA was prepared with a PureLink RNA Mini Kit (Life Technologies) with an additional DNase treatment step (PureLink DNase (Life Technologies)) and then reverse transcribed to cDNA with RevertAid H Minus Reverse Transcriptase and $500 \mu \mathrm{M}$ random pentadecamers (MWG). Quantitative PCR was performed with a CFX96 detection system (Bio-Rad) with Maxima SYBR Green master mix (Fermentas) and QuantiTect Primer Assays (Qiagen) and the following primers for mouse Gapdh, 5'-ACGGGAAGCTCACTGGCATGGCCTT-3' (forward), 5'-CATGAG GTCCACCACCCTGTTGCTG-3' (reverse). The primers for analysis of pre-mRNA were as follows: Dbp, $5^{\prime}$-GCTCTGAGAACGCAGACCTC- $3^{\prime}$ (forward), 5'-AGGTCATTAGCACCTCCACG-3' (reverse); Rev-Erbo, 5'-CCCTCCCCTTGTGTTCTCTT- $3^{\prime}$ (forward), 5'-CTGCCATTGGAGCT GTCACT-3' (reverse); Bmall, 5'-TGTCGCACATCTGTATTGACG-3' (forward), $5^{\prime}$-CTTCCATGAGGGTCATCTTTGT-3' (reverse); Pck1, $5^{\prime}$-TTCTC TGGCCTGCAACTTTC-3' (forward), $5^{\prime}$-TTGTCTTCACTGAGGTGCCA- ${ }^{\prime}$ (reverse); G6pc, 5'-GGCTGATGCCTTGACAGTCT-3' (forward), 5'-AG TATACACCTGCTGCGCCC-3' (reverse); Lpl, 5' $5^{\prime}$-TCATTTCACATAGATG CTTGCC-3' (forward), 5'-GGGCACCCAACTCTCATACA-3' (reverse); Cyp7a1, 5'-CCCCCATATGTCTCCTTCCA-3' (forward), 5'-TAAATGGCAT TCCCTCCAGA- $3^{\prime}$ (reverse). The transcript levels for each gene were normalized to those of Gapdh or Hprt and evaluated according to the $2^{-\Delta \Delta \mathrm{Ct}}$ method $^{62}$.

Carbon monoxide gas treatment. Cells were cultured in sodium bicarbonateand phenol red-free DMEM (Sigma-Aldrich) containing 10\% FCS, $100 \mathrm{U} / \mathrm{ml}$ penicillin, $100 \mu \mathrm{g} / \mathrm{ml}$ streptomycin and $25 \mathrm{mM}$ HEPES. For bioluminescence recordings (Fig. 5c), reporter cells were seeded in 35-mm dishes and incubated with $250 \mu \mathrm{M}$ D-luciferin in light- and gas-tight aluminum boxes, including single photomultiplier tubes (Hamamatsu Photonics). For gene-expression studies (Fig. 3b and Supplementary Fig. 2) cells were seeded in 24-well plates and incubated in gas mixtures of $6 \%$ carbon monoxide ( $99.97 \%$; Linde AG) with $94 \%$ air or 6\% nitrogen (99.99\%; Linde AG) with $94 \%$ air generated with DIGAMIX gas-mixing pumps (H. Wösthoff). These pumps have the highest accuracy for preparing high-quality calibration gas mixtures from pure gases. The gas mixtures were humidified to $100 \%$ and passed with a flow of $20-30 \mathrm{ml} / \mathrm{min}$ through gastight tubes, and aluminum or Plexiglas boxes incubated at $37^{\circ} \mathrm{C}$. According to Henry's law, $6 \% \mathrm{CO}$ results in an $\sim 50 \mu \mathrm{M} \mathrm{CO}$ concentration in aqueous solution at $37^{\circ} \mathrm{C}$ (ref. 63).

Carbon monoxide-releasing molecules (CORMs). CORMs (tricarbonyldichl ororuthenium(II) dimer; $\left[\mathrm{Ru}(\mathrm{CO})_{3} \mathrm{Cl}_{2}\right]_{2}$ ) or iCORMs (ruthenium(III) chloride; $\mathrm{RuCl}_{3}$ ) were purchased from Sigma-Aldrich and dissolved in a mixture of DMSO and ethanol. $1 \mathrm{~mol} \mathrm{CORMs}$ dissociates into $4 \mathrm{~mol} \mathrm{CO}$. We used $400 \mu \mathrm{M} \mathrm{CO}$ in these experiments, a concentration eight times higher than $6 \% \mathrm{CO}(50 \mu \mathrm{M})$; however, in contrast to that from constant gas treatment, CO from CORMs volatizes very rapidly, and only an undefined concentration reaches the cells.

Glucose production assay. $48 \mathrm{~h}$ after cell attachment, hepatocytes were synchronized with $1 \mu \mathrm{M}$ dexamethasone (Dex) for $1 \mathrm{~h}$, washed twice with PBS and incubated overnight with phenol red-free DMEM containing $5 \mathrm{mM}$ glucose, $0.5 \%$ FBS and $100 \mathrm{nM}$ glucagon. The next morning, cells were washed twice Then the medium was replaced with phenol red-free and glucose-free medium supplemented with $2 \mathrm{mM}$ pyruvate, $20 \mathrm{mM}$ lactate and $100 \mathrm{nM}$ glucagon for $5 \mathrm{~h}$. The glucose concentration in the medium was determined with an Amplex Red Glucose Assay Kit (Invitrogen) according to the manufacturer's instructions. Total protein content was determined with the bicinchoninic acid (BCA) method (Thermo Scientific) for normalization. 
Microarray experiments. Primary hepatocytes from $\mathrm{Ho}-\mathrm{1}^{-/-}$14- to 16 -week-old male mice or their wild-type littermates with or without additional $\mathrm{Ho}-2$ depletion were synchronized with dexamethasone. $24 \mathrm{~h}$ after synchronization, total RNA was isolated with a PureLink RNA Mini Kit (Life Technologies) with an additional DNase treatment step (PureLink DNase (Life Technologies)). The integrity and amount of total RNA were analyzed with an Agilent 2100 Bioanalyzer. Synthesis of cDNA, amplification, labeling and hybridization were performed by the Labor für Funktionelle Genomforschung (LFGC) with GeneChip Mouse Gene 1.0 ST arrays (Affymetrix).

Bioinformatics analysis. All steps of the bioinformatics analysis were performed in R 2.15.0 (http://www.R-project.org/). Raw data from Affymetrix arrays were preprocessed and RMA normalized with the 'affy' package ${ }^{64}$. Differentially expressed probe sets were identified with an empirical Bayes approach implemented in the 'limma' package ${ }^{65}$. A minimum expression value of 6 on at least two arrays was set as a threshold. The false discovery rate was set to 0.05 , and a minimum fold change of 1.5 was assumed between two experimental groups. Selected probe sets were clustered into six groups by application of a $k$-means algorithm to the scaled data. To ensure a robust reproducible solution, 100 random start sets for the cluster centers were used. Subsequently, probe sets were mapped to the associated genes. Identification of GO terms with an over-representation of associated genes in the clusters was performed with a classical Fisher test implemented in the package 'topGO' (version 2.10.0; http://www.bioconductor. org/packages/release/bioc/html/topGO.html). Because of the high redundancy of the GO hierarchy, no adjustment for multiple testing was done. Overlaps with data sets from Hughes et al. ${ }^{16}$ and Rey et al. ${ }^{17}$ were analyzed with the Fisher test with a multiple-testing Bonferroni correction of the $P$ values. Circadian transcripts from Hughes et al. ${ }^{16}$ were identified after fitting of the array data to a cosine-model with a Bonferroni-corrected $P$ value $<0.05$, a minimal magnitude of six and a minimal amplitude of 0.5 . E-box motifs from Rey et al. ${ }^{17}$ were used to calculate the TRAP scores ${ }^{66}$ genome wide for all promoter regions from $-1,000$ to $1,000 \mathrm{bp}$ relative to the potential transcription start site ${ }^{67}$. The maximum number of background sets was randomly sampled without replacement in the same sizes as the foreground sets (genes in clusters), and the scores were tested for higher values in the foreground sets with single-sided Wilcoxon rank-sum tests. All $P$ values were $\log _{10}$-transformed for plotting, and the geometric mean was calculated.

Statistics, sample size and conditions. Statistical test are described in the respective figure legends or in the section above. Sample sizes were not predetermined by statistical methods, and experiments were not randomized or conducted blind to conditions.
Data availability. The microarray data of this study have been deposited in the NCBI Gene Expression Omnibus (GEO) database and are accessible through GEO series accession number GSE55448. Source data for all other experiments are available on request.

49. Sandrelli, F. et al. A molecular basis for natural selection at the timeless locus in Drosophila melanogaster. Science 316, 1898-1900 (2007).

50. Bischof, J., Maeda, R.K., Hediger, M., Karch, F. \& Basler, K. An optimized transgenesis system for Drosophila using germ-line-specific phiC31 integrases. Proc. Natl. Acad. Sci. USA 104, 3312-3317 (2007).

51. Renn, S.C., Park, J.H., Rosbash, M., Hall, J.C. \& Taghert, P.H. A pdf neuropeptide gene mutation and ablation of PDF neurons each cause severe abnormalities of behavioral circadian rhythms in Drosophila. Cell 99, 791-802 (1999).

52. Sepp, K.J., Schulte, J. \& Auld, V.J. Peripheral glia direct axon guidance across the CNS/PNS transition zone. Dev. Biol. 238, 47-63 (2001).

53. Dimitroff, B. et al. Diet and energy-sensing inputs affect TorC1-mediated axon misrouting but not TorC2-directed synapse growth in a Drosophila model of tuberous sclerosis. PLoS One 7, e30722 (2012).

54. Chen, K.F., Peschel, N., Zavodska, R., Sehadova, H. \& Stanewsky, R. QUASIMODO, a novel GPI-anchored zona pellucida protein involved in light input to the Drosophila circadian clock. Curr. Biol. 21, 719-729 (2011).

55. Levine, J.D., Funes, P., Dowse, H.B. \& Hall, J.C. Signal analysis of behavioral and molecular cycles. BMC Neurosci. 3, 1-25 (2002).

56. Wilson, C.G. et al. Liver-specific overexpression of pancreatic-derived factor (PANDER) induces fasting hyperglycemia in mice. Endocrinology 151, 5174-5184 (2010).

57. Gekakis, N. et al. Role of the CLOCK protein in the mammalian circadian mechanism. Science 280, 1564-1569 (1998).

58. Maier, B. et al. A large-scale functional RNAi screen reveals a role for CK2 in the mammalian circadian clock. Genes Dev. 23, 708-718 (2009).

59. Locke, J.C.W. et al. Extension of a genetic network model by iterative experimentation and mathematical analysis. Mol. Syst. Biol. 1, 2005.0013 (2005).

60. Klemz, R., Mashreghi, M.F., Spies, C., Volk, H.D. \& Kotsch, K. Amplifying the fluorescence of bilirubin enables the real-time detection of heme oxygenase activity. Free Radic. Biol. Med. 46, 305-311 (2009).

61. Ripperger, J.A. \& Schibler, U. Rhythmic CLOCK-BMAL1 binding to multiple E-box motifs drives circadian Dbp transcription and chromatin transitions. Nat. Genet. 38, 369-374 (2006)

62. Livak, K.J. \& Schmittgen, T.D. Analysis of relative gene expression data using realtime quantitative PCR and the $2^{-\Delta \Delta C T}$ method. Methods 25, 402-408 (2001).

63. Lide, D.R. CRC Handbook of Chemistry and Physics: a Ready-Reference Book of Chemical and Physical Data (CRC Press, 1995).

64. Gautier, L., Cope, L., Bolstad, B.M. \& Irizarry, R.A. affy: analysis of Affymetrix GeneChip data at the probe level. Bioinformatics 20, 307-315 (2004).

65. Smyth, G.K. in Bioinformatics and Computational Biology Solutions Using $R$ and Bioconductor (eds. Gentleman, R., Carey, V., Huber, W., Irizarry, R. \& Dudoit, S.) 397-420 (Springer, 2005)

66. Roider, H.G., Kanhere, A., Manke, T. \& Vingron, M. Predicting transcription factor affinities to DNA from a biophysical model. Bioinformatics 23, 134-141 (2007).

67. Flicek, P. et al. Ensembl 2012. Nucleic Acids Res. 40, D84-D90 (2012). 